\title{
Spent Nuclear Fuel System Dynamic Stability under Normal Conditions of Transportation
}

\author{
Hao Jiang and Jy-An John Wang ${ }^{*}$ \\ Materials Science and Technology Division, Oak Ridge National Laboratory \\ One Bethel Valley Road, Oak Ridge, Tennessee 37831 \\ *Tel: 1-865-574-2274; Fax: 1-865-574-6098; email: wangja@ornl.gov
}

\begin{abstract}
In a horizontal layout of a spent nuclear fuel (SNF) assembly under normal conditions of transportation (NCT), the fuel assembly's skeleton formed by guide tubes and spacer grids is the primary load bearing structure for carrying and transferring the vibration loads within an SNF assembly. Therefore, the integrity of guide tubes and spacer grids will dictate the vibration amplitude/intensity of the fuel assembly during transport, and must be considered when designing multipurpose purpose canister (MPC) for safe SNF transport. This paper investigates the SNF assembly deformation dynamics during normal vibration mode, as well as the transient shock mode inside the cask during NCT. Dynamic analyses were performed in the frequency domain to study frequency characteristic of the fuel assembly system and in the time domain to simulate the transient dynamic response of the fuel assembly.

To further evaluate the intensity of contact interaction induced by the local contacts' impact loading at the spacer grid, detailed models of the actual spring and dimples of the spacer grids were created. The impacts between the fuel rod and springs and dimples were simulated with a $20 \mathrm{~g}$ transient shock load. The associated contact interaction intensities, in terms of reaction forces, were estimated from the finite element analyses (FEA) results. The bending moment estimated from the resultant stress on the clad under $20 \mathrm{~g}$ transient shock can be used to define the loading in Cyclic Integrated Reversible-Bending Fatigue Tester (CIRFT) vibration testing for the equivalent condition. To estimate the damage potential of the transient shock to the SNF vibration lifetime, drop tests were performed on the CIRFT specimens. FEA was used to investigate the contact reaction at CIRFT test samples during impact loading induced by drop tests, and the result was compared with that from a $20 \mathrm{~g}$ acceleration transient shock load.
\end{abstract}

Key words: Spent nuclear fuel assembly, dynamic, normal condition of transport

\section{INTRODUCTION}

This research supports the US Department of Energy (DOE) Used Fuel Disposition Campaign modeling, simulation, and experimental integration research, development, and deployment (RD\&D) plan for spent nuclear fuel (SNF) integrity and structural performance under normal conditions of transportation (NCT) [1]. The PWR fuel assembly skeleton [2], as shown in FIGURE 1, is formed by guide tubes and spacer grids designed to constrain fuel rods during a reactor operation. In a vertical service setup, the skeleton is subjected to vibration loads induced by fluid dynamics, and the rods' dead weight is transmitted through the spacer grids to the guide tubes during reactor operation. When the SNF assembly is in a horizontal orientation for NCT, the skeleton formed by the guide tubes and spacer grids becomes the primary load-bearing system that carries and transfers the vibration loads within an SNF assembly. This includes interaction of forces between the SNF assembly and the canister basket walls.

This manuscript has been authored by UT-Battelle, LLC, under Contract No. DE-AC0500OR22725 with the U.S. Department of Energy. The United States Government retains and the publisher, by accepting the article for publication, acknowledges that the United States Government retains a non-exclusive, paid-up, irrevocable, world-wide license to publish or reproduce the published form of this manuscript, or allow others to do so, for the United States Government purposes. The Department of Energy will provide public access to these results of federally sponsored research in accordance with the DOE Public Access Plan (http://energy.gov/downloads/doe-public-access-plan). 
In an EPRI Synthesis Report-1015048 [3], the plastic collapse of the spacer grids and the breakage of the guide tubes were considered as the two modes for evaluating fuel assembly damage. Therefore, the integrity of guide tubes and spacer grids during transport requires full attention since it will affect the dynamic load transmitting mechanism within the fuel assembly and will consequently dictate the multipurpose purpose canister (MPC) design concept to ensure safe SNF transport.

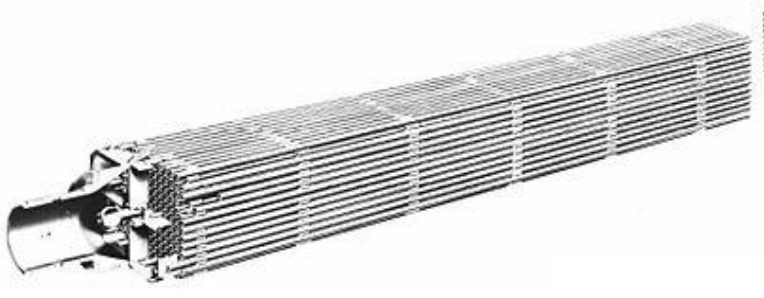

Fuel assembly
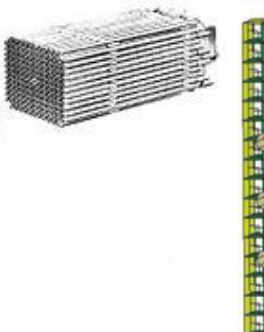

Guide tubes and spacer grids [2]

FIGURE 1. Schematic of $17 \times 17$ PWR fuel assembly.

Random vibration registered at the SNF transport cask, which is excited from the railcar bed, provides the external loading driver to vibrate the SNF assembly. In addition to this external vibration driver, the fuel assembly also registers internal transient shocks resulting from the dynamic interactions among the fuel assembly components inside the cask. These components include the skeleton, fuel rods, and canister basket walls.

Their dynamic interactions can significantly increase the high-rate impact loading frequency within fuel assembly components during NCT. Sandia National Laboratories (SNL) registered maximum 22 g peak vertical vibration acceleration at mid span of the surrogate rod adjacent to the spacer grid from the accelerometer reading during the truck transportation test [4]. In contrast, the maximum vertical acceleration of $5.6 \mathrm{~g}$ was registered on the top of transport basket at mid-span.

The aged or fatigued skeleton has the potential to increase the gap population and intensity within the fuel assembly system. The consequence of increased gap potential is the increase in contact impact loading intensity between the fuel rods, the skeleton, and the basket wall. This can also enhance the probability of SNF system resonance vibration potential for a high burn-up fuel assembly. The contact interactions between fuel rods, spacer grids, and basket wall can also increase fuel rod transient shock load frequencies. Thus, external cask vibration needs to be mitigated, along with internal amplification from the fuel assembly system vibration and its contact-interaction transient shocks. System damping may need to be increased to reduce vibration intensity. Such mitigations will eventually impact the design of the canister system.

A multi-laboratory team consisting of researchers at Pacific Northwest National Laboratory (PNNL), Idaho National Laboratory (INL), Sandia National Laboratories (SNL), and Oak Ridge National Laboratory (ORNL) are developing a simulation approach to evaluate loading and associated mechanical responses of SNF rods and key structural components during NCT [2, 5]. The work is focused on structural performance evaluation of Westinghouse Electric $17 \times 17$ Optimized Fuel Assembly pressurized water reactor fuel assemblies. The assemblies have three levels: the cask level [6], the fuel assembly level [7], and the fuel rod level [8]. This was simulated with different emphasis on each level. Idaho National Laboratory developed models of the fuel rod level, which consisted of pellet numbers ranging from 2-54 for different study purposes [9]. EPRI report 1015049 [10] describes the response of a high-burnup fuel assembly to dynamic forces resulting from a one-foot drop onto a rigid surface. Each rod in the fuel assembly was modeled as a beam. The analysis emphasized rod-to-rod interaction forces, 
bending moments, etc. Ross et al. [11, 12] evaluated frequency domain and time domain of a realistic cradle supporting a PWR cask and a concrete block of equivalent mass and footprint.

This present research focuses on the targeted SNF subassembly deformation dynamics that can occur during normal vibration mode, as well as the cask's internal transient shock mode during NCT. The two fuel rods with a guide tube, the spacer grids (FIGURE 1) at the bottom edge of full fuel assembly, and the partial basket wall were all modeled to investigate interaction of fuel assembly components, as well as interaction against the basket wall or the spacer grids. Each fuel rod was modeled with the actual dimensions of 240 fuel pellets in a 3.658-m-long fuel cladding. Dynamics analyses were performed in the frequency domain to gain a better understanding of the frequency characteristics of the fuel assembly system and in the time domain to simulate the transient dynamic response of the fuel assembly. FEA simulation results are used to determine the stress and strain states of subassembly model components such as fuel rods, the guide tube, spacer grids, nozzles, and the basket wall under vibration loading during normal vibration conditions and transient shocks during NCT. The resulting stress/strain data can be used in future fuel assembly component failure analyses. The contact interactions between system components during transient dynamic simulation were investigated.

Detailed models of the spring and dimples from the actual spacer grids were created to further evaluate the intensity of contact interaction induced by the local contacts' impact loading at the spacer grid. The impacts between fuel rod, springs, and dimples were simulated with a $20 \mathrm{~g}$ transient shock load. The associated contact interaction intensities, in terms of reaction forces, were estimated from the FEA results. Based on the resultant stress induced by a $20 \mathrm{~g}$ acceleration, the corresponding bending moment was also determined. This equivalent bending loading will then be applied to SNF rod in a cyclic integrated reversible-bending fatigue tester (CIRFT) [13-15] to determine the associated SNF fatigue life under the periodic occurrence of such contact transient shocks. To estimate the damage potential of the transient shock to the SNF normal vibration lifetime, drop tests were performed on the CIRFT specimens. The combined drop-impact and CIRFT test results show reduced fatigue lifetime from SNF CIRFT specimens. FEA was used to investigate the contact reaction at CIRFT test samples during impact loading induced by drop tests, and the result was compared with the result from a $20 \mathrm{~g}$ acceleration transient shock load.

\section{SNF ASSEMBLY DYNAMIC SIMULATION}

The FEA modeling effort focused on the SNF assembly in a transportation mode in a horizontal setup. The targeted SNF assembly shown in FIGURE 1 was simplified using a three-dimensional ABAQUSexplicit transient finite element model (FEM) shown in FIGURE 1 Error! Reference source not found.. This simplification allows for a meaningful, practical investigation of the SNF rod's dynamic response and the SNF system components' interactions during NCT. The skeleton of the SNF assembly consists of guide tubes and spacer grids, which serve as the primary structural components carrying the vibration loading induced by the railcar bed and transferred from outside the transport cask. The 240 fuel pellets, with lengths of $0.0152 \mathrm{~m}$, were modeled in a 3.658-m-long fuel cladding in which pellets contact each other at their ends. In one fuel rod, there are 239 contact pairs within the fuel pellets. FIGURE 3 shows the contact pairs in a section of the partial model.

There were several key assumptions and primary simplifications made in the development of the targeted subassembly model. Two fuel rods are modeled with a guide tube at the bottom edge of full fuel as highlighted in FIGURE 1. The guide tube is on top of two fuel rods as illustrated in FIGURE 2. Each fuel rod has 240 fuel pellets and a cladding. The guide tube and two fuel rods are connected with eight spacers, including top and bottom Inconel spacers, as well as six intermediate $\mathrm{Zr}-4$ spacers. The top and 
bottom nozzles are simplified as two end plates to eliminate unnecessary geometric details. A long rectangular plate is modeled to represent the basket floor, and is assumed to be part of the basket enclosure to interact with the fuel rod assembly from the bottom, as shown in FIGURE 2Error! Reference source not found.

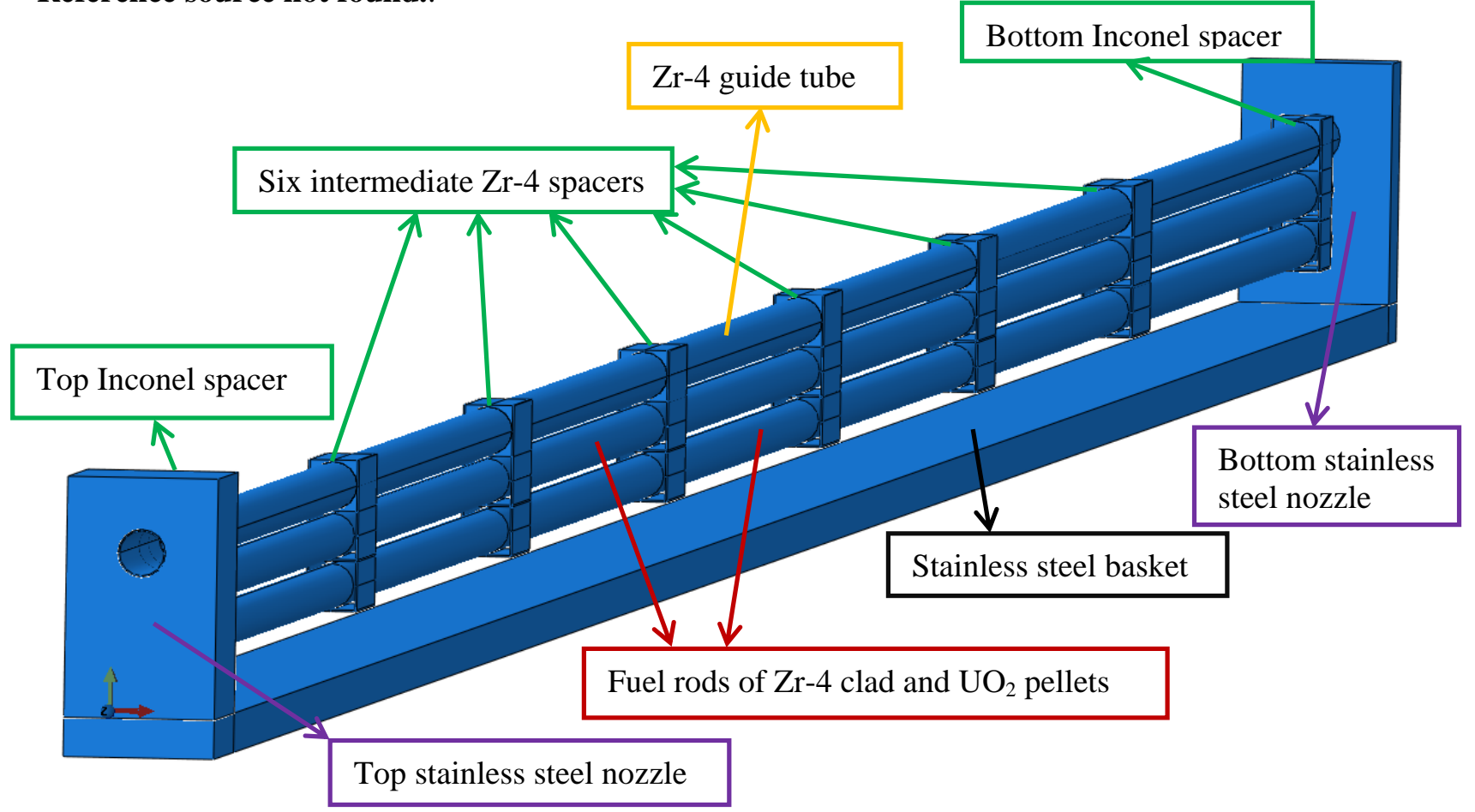

FIGURE 2. SNF subassembly model for normal transportation evaluation.

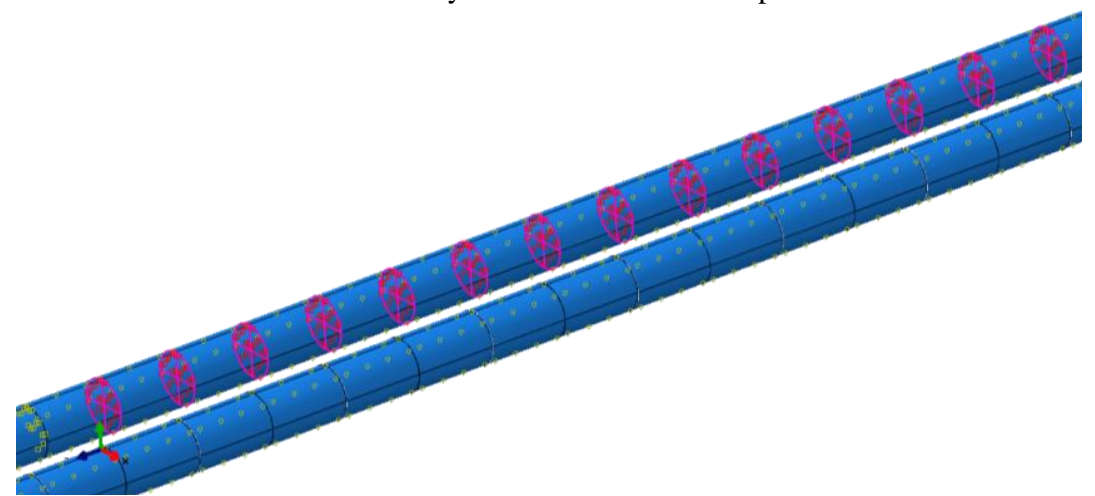

FIGURE 3. Section of the partial model shows pellets contact each other

The spacer grids are modeled as simplified box structures, and the spacer grid leaf springs/dimples are modeled as translational springs. Fuel rods are connected to spacer grids through the springs/dimples, as shown in FIGURE 4. The guide tube is rigidly connected to the spacer grids via the tie constraint in ABAQUS. The guide tube is connected to the nozzles via the tie, and the nozzles are tied to the basket to properly transfer the dynamic loading. The geometry of the fuel subassembly model is taken primarily from dimensions provided in DOE's Used Nuclear Fuel Loading and Structural Performance Under Normal Conditions of Transport-Demonstration of Approach and Results of Used Fuel Performance Characterization [2]. This fuel subassembly model retains the proper mass and inertial properties for dynamic simulation. 

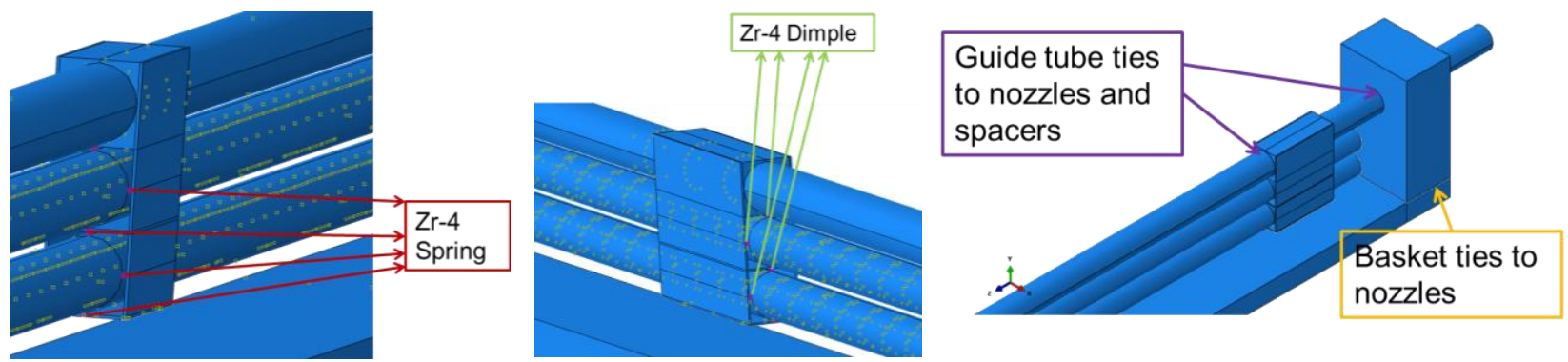

FIGURE 4. Spacer grid leaf spring/dimples modeled as translational springs and tie constraint at boundaries.

All components are meshed with 8-node linear hexahedral brick elements in the SNF fuel subassembly model. The fuel rods are supported by two sets of translational springs in every slot: one set of leaf springs and one set of dimples. Special care is taken to stabilize the dynamic calculation, including mesh distortion control, severe distorted element deletion, reduced integration and hourglass control, etc. However, a long solution time for dynamic simulation is still anticipated since a very small time increment is required to converge the solution. Furthermore, the model consists of roughly 130,000 nodes and 80,000 elements, which also result in a long solution time.

Contact is implemented throughout the fuel assembly between interacting components. Surface-to-surface contacts between the guide tube and fuel rod, between spacer grids and basket floor, and between fuel rod and basket floor are defined when they come to touch each other. The friction coefficient is reasonably assumed as 0.3 at contact surfaces.

As shown in FIGURE 2, fuel pellets are made of $\mathrm{UO}_{2}$, while fuel cladding, the intermediate spacer grids, and the guide tube are made of Zircaloy-4. The top and bottom spacer grids are made of Inconel, and the top and bottom nozzles and basket are made of stainless steel. The material properties of the fuel assembly's components are listed in TABLE 1. The leaf spring and dimple spring material is Zircaloy-4. The spring stiffness value for Zircaloy-4 leaf spring is $49 \mathrm{kN} / \mathrm{m}$, and the value for Zircaloy- 4 dimple is $126 \mathrm{kN} / \mathrm{m}[2]$.

TABLE 1. Mechanical properties of the fuel assembly.

\begin{tabular}{llcccc}
\hline & Material & $\begin{array}{c}\text { Young's modulus } \\
(\mathrm{GPa})\end{array}$ & $\begin{array}{c}\text { Poisson's } \\
\text { ratio }\end{array}$ & $\begin{array}{c}\text { Yield strength } \\
(\mathrm{MPa})\end{array}$ & $\begin{array}{c}\text { Density } \\
\left(\mathrm{kg} / \mathrm{m}^{3}\right)\end{array}$ \\
\hline Pellet & $\mathrm{UO}_{2}[16]$ & 201 & 0.32 & 2,146 & 10,970 \\
$\begin{array}{l}\text { Clad, } \\
\text { intermediate } \\
\text { spacer grids, } \\
\text { guide tube }\end{array}$ & Zircaloy-4 [17] & 91 & 0.33 & 906 & 6,560 \\
$\begin{array}{l}\text { Top and bottom } \\
\text { spacer grids }\end{array}$ & Inconel [18] & 211 & 0.284 & 987 & 8,193 \\
Basket, nozzles & Stainless steel [19,20] & 193 & 0.3 & 290 & 8,030 \\
\hline
\end{tabular}

\subsection{Modal Analysis}

Dynamics analysis of a vibrating system is often performed in the frequency domain because the dynamic response of structures depends on the frequency characteristic of the system, namely natural frequencies and mode shapes. Resonance occurs when the excitation frequency loaded on the structure is near its natural frequency, and it will significantly amplify the system dynamic response to the extent that it can 
cause structure failure. Modal analysis is used to determine the natural frequencies of the SNF subassembly model and the dominant modes. Such results provide a better understanding of frequency characteristic of the fuel assembly systems.

A modal analysis of the SNF subassembly model was conducted using ABAQUS linear perturbation frequency code in which the system is modeled with a simply supported structure. The first 6 mode shapes and associated natural frequencies are shown in FIGURE 5. The guide tube and fuel rods are mostly synchronized (i.e., within the same phase), while the basket floor vibrates within a difference phase. The fundamental mode of this subassembly model has a natural frequency of $\sim 2 \mathrm{~Hz}$, which is considerably lower than that of the cradle/cask system models with full fuel assembly weights, as described in Ross 2015 [11,12].

When higher vibration modes occur, as shown in FIGURE 6, system components such as the guide tube and fuel rods are no longer in the same phase. Local vibration motions are involved with different components, and in some modes (e.g., modes 17 and 18), the fuel rod vibrations become dominant.

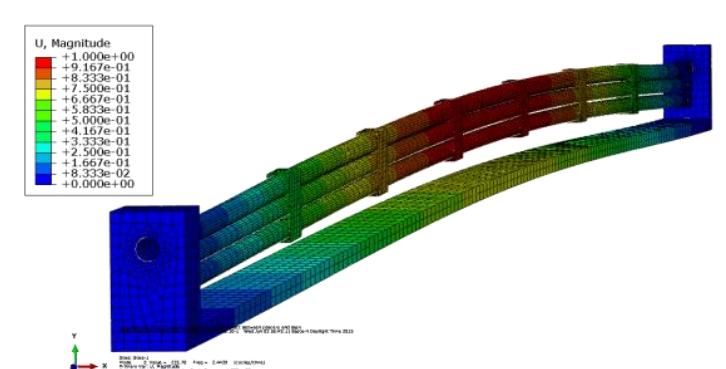

Mode-1: $2.444 \mathrm{~Hz}$

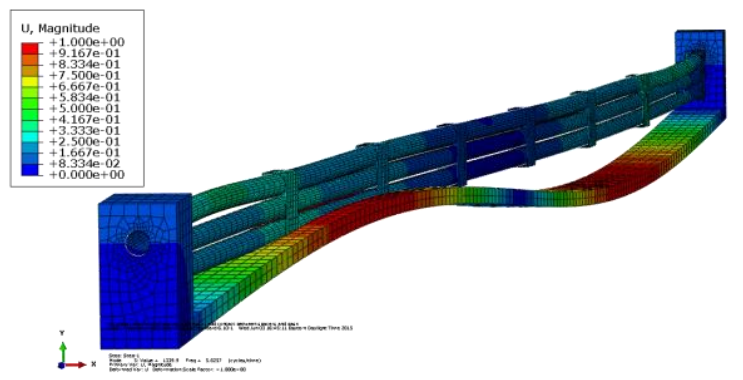

Mode-3: $5.826 \mathrm{~Hz}$

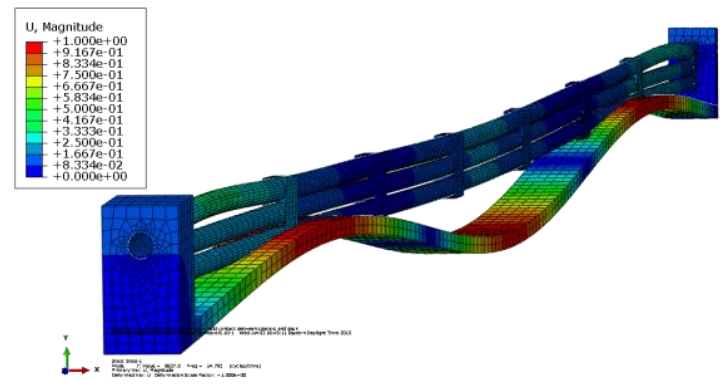

Mode-5: $14.792 \mathrm{~Hz}$

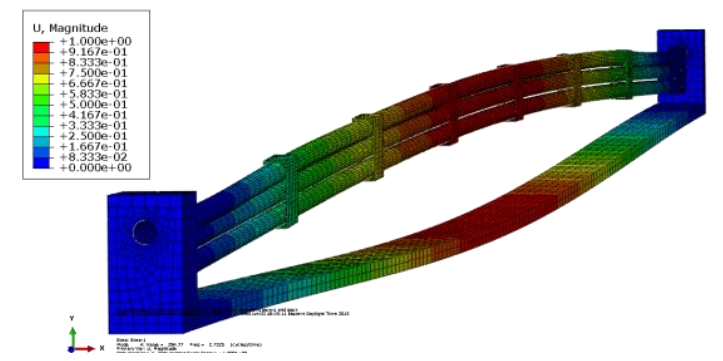

Mode-2: $2.733 \mathrm{~Hz}$

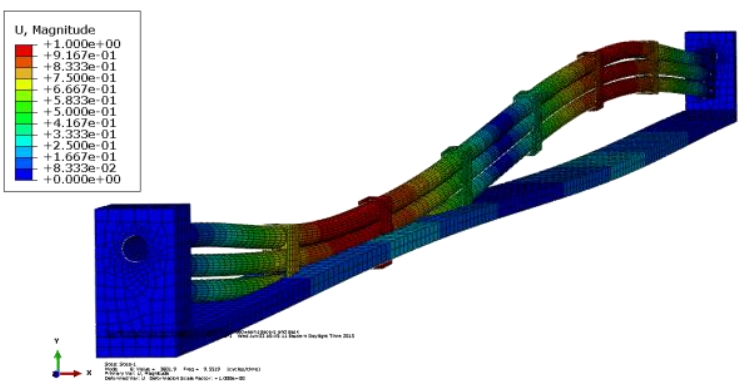

Mode-4: $9.552 \mathrm{~Hz}$

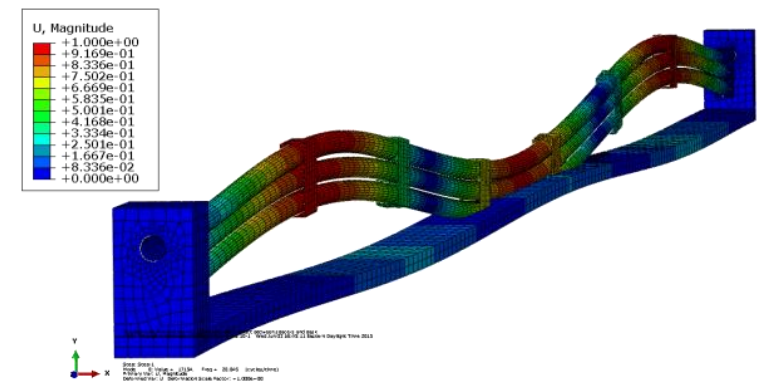

Mode-6: $20.845 \mathrm{~Hz}$

FIGURE 5. The first 6 modes and the associated natural frequencies of the fuel subassembly model. 


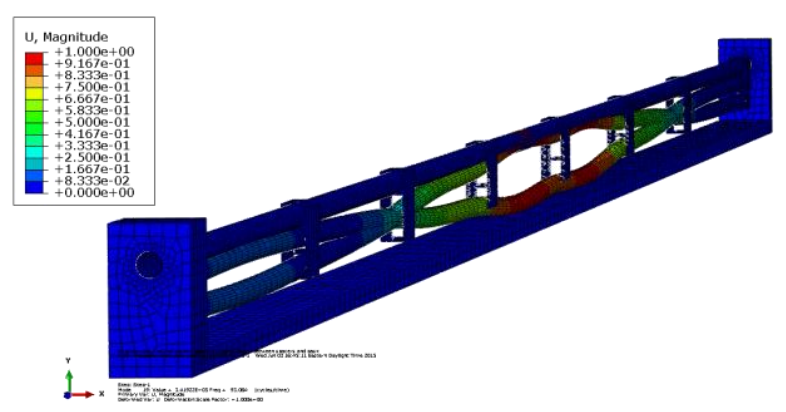

Mode-17: $93.064 \mathrm{~Hz}$

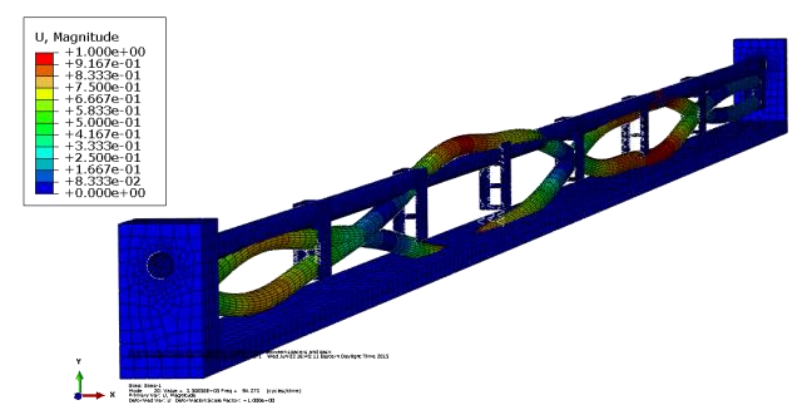

Mode-18: $94.273 \mathrm{~Hz}$

FIGURE 6. Higher modes and associated natural frequencies of the fuel assembly sub-model.

Modal analysis results of the SNF fuel subassembly model are summarized in TABLE 2. The natural frequencies for the mode are listed up to mode 51, and the associated participation factor and effective mass are evaluated accordingly. The relative strength of each mode is ranked by the participation factors, which are normalized and listed as ratio values. The participation factor with the strongest mode is assigned a value of 1.0, and the other participation factors are normalized to that value. The participation of effective mass in the deformation of the mode shape is another way to indicate the strength of modes. Larger mass indicates a stronger mode. The participation factor and effective mass correspond to each other. The strongest mode is the first mode's frequency of $2.4 \mathrm{~Hz}$ as shown in TABLE 2. As shown in FIGURE 5, the modes containing odd numbers of half sine wave have higher participation factor ratios. For example, mode 5's frequency of $14.8 \mathrm{~Hz}$ and mode 6's frequency of $20.8 \mathrm{~Hz}$ have $3 \mathrm{half}$ sine wave shapes in the basket floor, the guide tube, and the fuel rods, respectively, where the participation factor ratio is in the second and third place. As discussed in Ross 2015 [11,12], a modal analysis was performed for a realistic cradle/cask system model and a concrete block model of equivalent mass. The results show that the strongest mode for the cradle/cask system is at frequency of $52.4 \mathrm{~Hz}$. In the case of the concrete block model, the strongest mode is $318.1 \mathrm{~Hz}$, which is as high as the frequency of mode 49 for the fuel subassembly model. The modal analysis indicates that system models with different structure level setups and different associated boundary conditions reveal significantly different natural frequency characteristics.

TABLE 2. Modal analysis of SNF assembly sub-model.

\begin{tabular}{cccc}
\hline Mode number & Frequency & $\begin{array}{c}\text { Participation } \\
\text { factor ratio }\end{array}$ & $\begin{array}{c}\text { Effective mass } \\
(\mathbf{N ~ s} \mathbf{~} \mathbf{m})\end{array}$ \\
\hline 1 & 2.4 & 1.000 & $1.435 \mathrm{E}+01$ \\
2 & 2.7 & 0.162 & $5.026 \mathrm{E}-01$ \\
3 & 5.8 & 0.004 & $2.182 \mathrm{E}-04$ \\
4 & 9.6 & 0.004 & $1.121 \mathrm{E}-04$ \\
5 & 14.8 & 0.381 & $1.890 \mathrm{E}+00$ \\
6 & 20.8 & 0.236 & $4.422 \mathrm{E}-01$ \\
7 & 22.7 & 0.011 & $1.816 \mathrm{E}-03$ \\
8 & 36.1 & 0.076 & $5.003 \mathrm{E}-02$ \\
9 & 37.7 & 0.221 & $6.944 \mathrm{E}-01$ \\
10 & 50.3 & 0.026 & $1.012 \mathrm{E}-02$ \\
11 & 55.5 & 0.182 & $2.485 \mathrm{E}-01$ \\
12 & 71.0 & 0.163 & $3.266 \mathrm{E}-01$ \\
13 & 75.9 & 0.001 & $1.126 \mathrm{E}-06$ \\
14 & 76.5 & 0.063 & $1.418 \mathrm{E}-02$
\end{tabular}




\begin{tabular}{llll}
15 & 90.1 & 0.026 & $7.667 \mathrm{E}-03$ \\
16 & 92.6 & 0.000 & $8.707 \mathrm{E}-08$ \\
17 & 93.1 & 0.000 & $7.455 \mathrm{E}-09$ \\
18 & 94.3 & 0.000 & $8.583 \mathrm{E}-08$ \\
$\ldots$ & & & \\
49 & 318.3 & 0.001 & $1.069 \mathrm{E}-06$ \\
51 & 345.1 & 0.014 & $1.074 \mathrm{E}-04$ \\
\hline
\end{tabular}

\subsection{Transient Dynamic Analysis}

The objective of the transient dynamic analysis of the fuel assembly sub-model was to determine the stress and strain states of subassembly model components in response to vibration loading of transient shock under NCT. Components included the fuel rods, guide tube, spacer grids, nozzles, and basket wall. The resulting stress/strain can be used in further component failure analyses. The contact interactions between fuel assembly system components during transient dynamic simulation were studied. The fuel subassembly model contains numerous contact conditions and represents itself as a highly nonlinear system. The transient dynamic analyses were performed using ABAQUS-explicit code in the time domain for nonlinear system evaluation.

There are 264 fuel rods contained in the spacer grid slots because there are 25 guide tubes for a 17 x 17 fuel assembly. The guide tube modeled at the edge of the full SNF assembly will roughly carry the gravity load of the neighboring 10 fuel rods through the spacer grids. A gravitational acceleration loading of $9.8 \mathrm{~m} / \mathrm{s}^{2}$ is applied to all components. The gravitation loads from the remaining 8 fuel rods are applied as the concentrated loads evenly distributed to eight spacer grids, as shown in FIGURE 7.

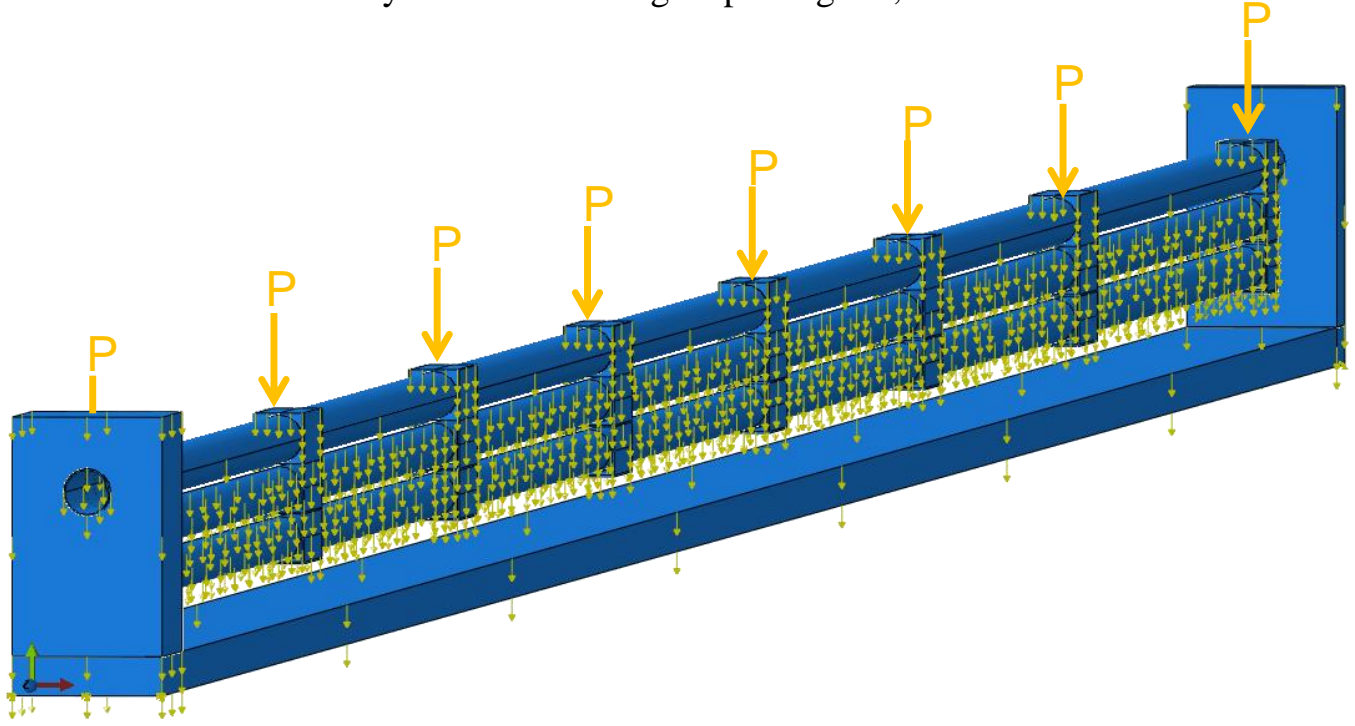

FIGURE 7. Gravity loads for the initial stage of transient dynamic simulation of the fuel subassembly model.

The developed dynamic simulation protocol consists of two stages. During the first stage, the basket wall is constrained, while the assembly (mainly the intermediate spacer grids) sags down due to the gravity load and comes to rest on the basket floor. During the second stage, the acceleration with a sine wave form or impulse signal to represent the normal vibration excitation or transient shock excitation that was applied to the basket and nozzles for transient dynamic analyses. In the second transient excitation stage, the constraint at the basket floor is removed, but the gravity loads are maintained for both stages of 
simulations. Therefore, the procedure for the subassembly model includes an initialization stage to establish gravity-induced inter-contacting followed by a transient dynamic loading stage. This procedure is similar to that provided in Adkins et al., Sept. 2013 [2].

The stress distribution (von Mises) and deformation of the SNF subassembly model after the initialization stage is shown in FIGURE 8. During the first stage, only gravity load is applied to the system, and the basket floor is assumed to be simply supported at the cask. Maximum stress of $287 \mathrm{MPa}$ occurs at the guide tube. Maximum stress level is below all the material yield strengths listed in TABLE 1, so no yielding is observed in the system. The guide tube and fuel rods sag in the middle sections as a result of dead weight loads. Close-up views show that intermediate spacer grids rested on the basket floor after the subassembly model assembly sagging stabilized.

The top and bottom spacer grids did not contact the basket floor. Maximum contact pressure occurs at an intermediate spacer grid touching the basket wall, as shown in FIGURE 8. Contact pressure level is relatively low because the fuel assembly gradually sags down to reach the basket floor, and the loading process was assigned as a ramp-up in conjunction with the quasi-static state loading condition during each loading interval.

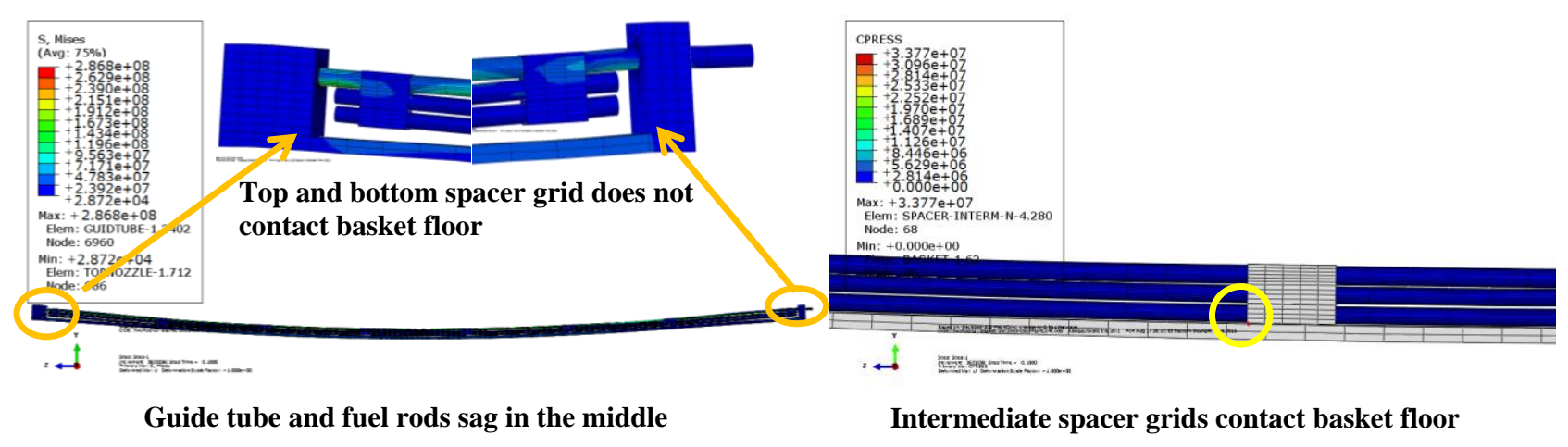

FIGURE 8. Dynamic response of the fuel assembly sub-model for the initialization stage.

The typical load-time history [21] registered by an accelerometer on a transport cask is shown in FIGURE 9(a). It illustrates transient shock signals superimposed on continuous vibration signals of NCT. This random vibration from the cask provides the external loading to the SNF assembly. Inside the cask, another form of transient shocks comes from the dynamic impact induced by dynamic contact interactions between the fuel assembly components during NCT. These components include the canister basket wall, guide tube, fuel rods, and spacer grids.

During the second stage, gravity loading remained, and the constraint on the basket wall was removed. The typical vibration load as acceleration-time history was applied to the basket and the top and bottom nozzles, exciting the SNF subassembly model under normal vibrations or transient shocks of NCT. The first case of transient dynamics analysis is to simulate normal vibrations during NCT. FIGURE 9(b) illustrates that a $0.5 \mathrm{~g}$ sine wave acceleration excitation was applied to the basket wall and the top and bottom nozzles of the fuel subassembly model. The sine wave has a frequency of $5 \mathrm{~Hz}$. 


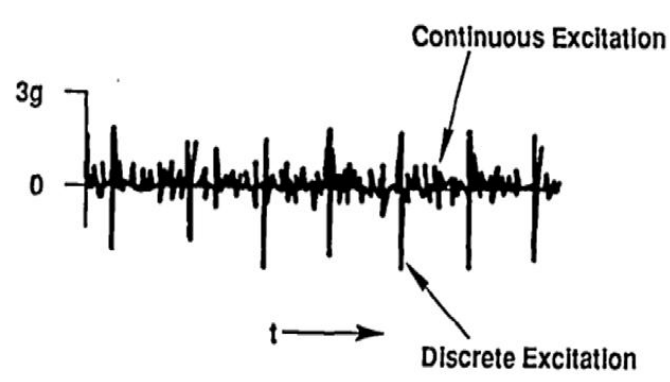

(a) Typical acceleration time history[21]

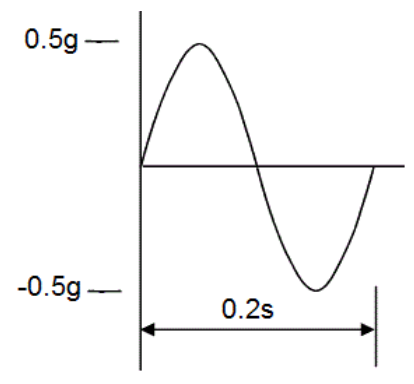

(b) A $0.5 \mathrm{~g}$ sine wave acceleration excitation

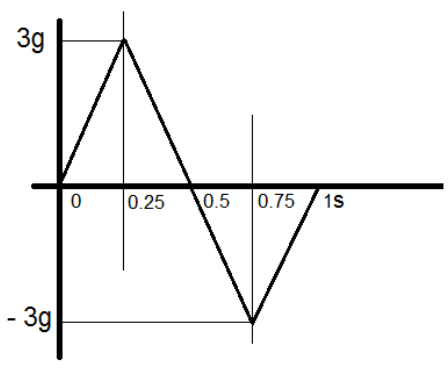

(c) A $3 g$ impulse acceleration excitation

FIGURE 9. The typical load-time history of a transport cask and excitation used to simulate the normal vibration of NCT.

The dynamic responses of the fuel subassembly model under a vibration of $0.5 \mathrm{~g}$ acceleration amplitude is shown in FIGURE 10(a). Stress level is higher than in the initial stage. After $0.5 \mathrm{~g}$ normal vibration excitation, the fuel rod, guide tube, spacer grids and basket wall deformed nonuniformly as compared to deformation during the initialization stage. Some intermediate spacer grids lost contact with the basket wall. According to von Mises stress distribution, besides the fuel pellets, the maximum stress occurs at the bottom Inconel spacer grid section where the material yields.

Guide tubes and spacer grids become load bearing members to carry the dead weight and transmit the dynamic loads in the fuel assembly system due to the horizontal orientation of the fuel assembly during SNF transport. Therefore, they are responsible for transferring the load within the fuel assembly during transport. The stress distributions in the guide tube are shown in FIGURE 10(b). The maximum stress in the guide tube reaches the yield strength. Yielding on the guide tube occurs at both top and bottom nozzle locations. FEA results indicate that the guide tube and spacer grids are at high risk of failure. The failure of the skeleton will increase the random vibration contact frequencies among the fuel rods, and it will also increase their vibration intensity. Therefore, the integrity of the guide tube and spacer grids can critically affect the vibration modes and the vibration intensity of fuel assembly during transport.
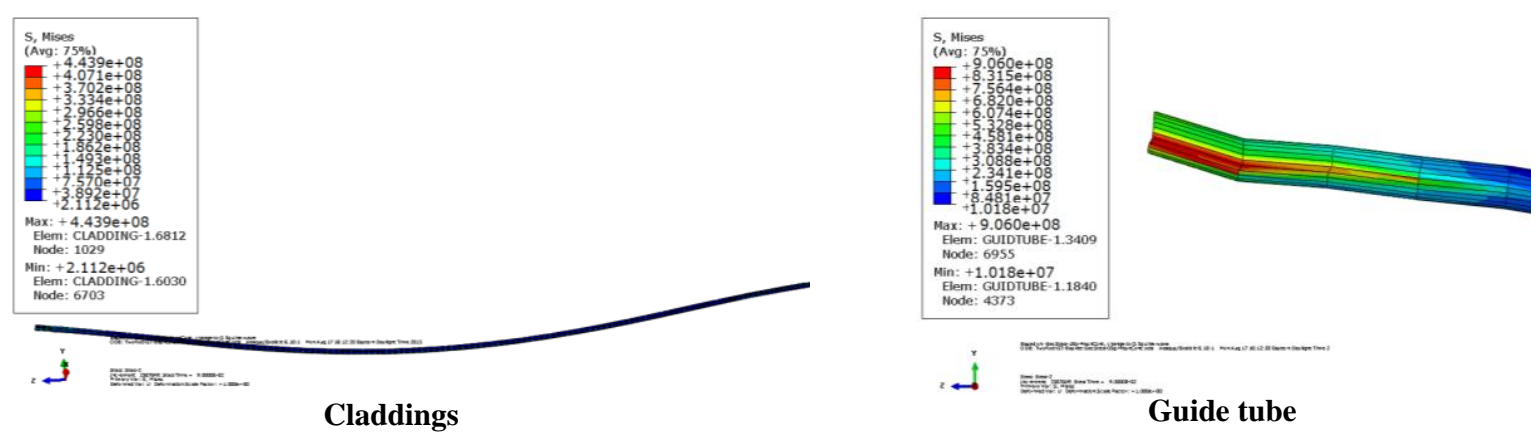

FIGURE 10. The dynamic response of fuel assembly and guide tube under $0.5 \mathrm{~g}$ sine wave acceleration excitation.

The stress distribution and deformation of other system components in the fuel subassembly model (including the fuel cladding, the basket floor, and the top and bottom nozzles) with reference to the dynamic response of vibration under $0.5 \mathrm{~g}$ sine wave acceleration excitation are shown in FIGURE 11 Under a $0.5 \mathrm{~g}$ simulated normal vibration load, the maximum stress of claddings is below the material yield strength, so the claddings remain intact. However, the basket wall and the top and bottom nozzles were yielded, possibly due to the transient shocks and/or impact load induced by the mutual contact interaction between the spacer grids, guide tube, and fuel rods. 

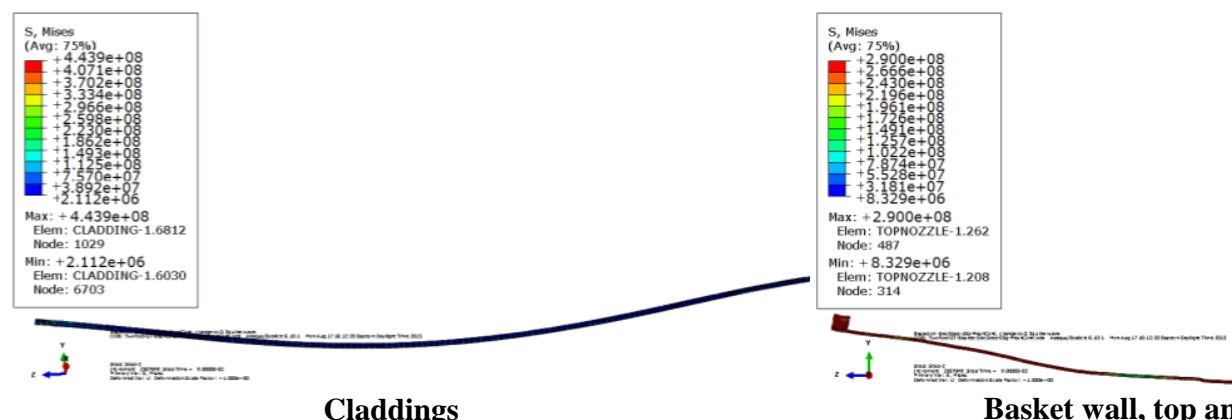

Basket wall, top and bottom nozzles

FIGURE 11. dynamic response of cladding, basket floor, top and bottom nozzles under $0.5 \mathrm{~g}$ sine wave acceleration excitation.

The maximum contact pressure of the SNF subassembly model under $0.5 \mathrm{~g}$ sine wave acceleration excitation is highlighted at the contact point of the spacer grid and the basket floor in FIGURE 12. When compared to the contact point in the initialization stage, the maximum contact pressure level is almost one order of magnitude higher. However, the maximum contact pressure is still under material yield strength.

The second phase of transient dynamics analysis is to study the dynamic response of the SNF subassembly model under transient shock loading during NCT. A periodic impulse excitation of $3 \mathrm{~g}$

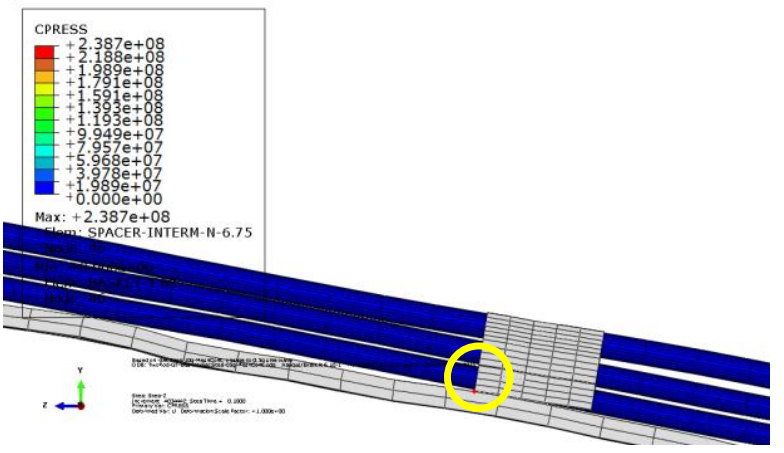

FIGURE 12. Maximum contact pressure under $0.5 \mathrm{~g}$ sine wave acceleration excitation resides at the contact point of the spacer grid and the basket floor. and period of $1 \mathrm{~s}$ was simulated as transient shock during transportation according to FIGURE 9(c). The resulting system dynamic responses within this loading cycle period are similar to the dynamic response of $0.5 \mathrm{~g}$ sine wave acceleration excitation. Yielding occurs at the bottom Inconel spacers during 3-g transient shock excitation. The guide tube also yields at the top and bottom nozzle locations. Therefore, the integrity of the spacer grids and guide tube are the primary concern under such transient shock loading during NCT. The dynamic response of claddings under 3-g transient shock excitation indicates that the maximum stress level is twice that of 0.5$\mathrm{g}$ sine wave acceleration excitation and is near the yield strength. The basket wall and the top and bottom nozzles also have regions that reach their associated yield strengths.

In the transient shock excitation case, the maximum contact pressure occurs at the location where the guide tube is in contact with the top fuel rod as shown in FIGURE 13 (marked with a yellow circle). The FEA result indicates that the maximum contact pressure level is high at this point. This type of dynamic contact interaction will inevitably form another pattern of transient shock loading within the fuel assembly system. Therefore, the contact interaction in the fuel assembly system, which can introduce transient shock loadings and impact SNF vibration integrity, needs more thorough investigation.

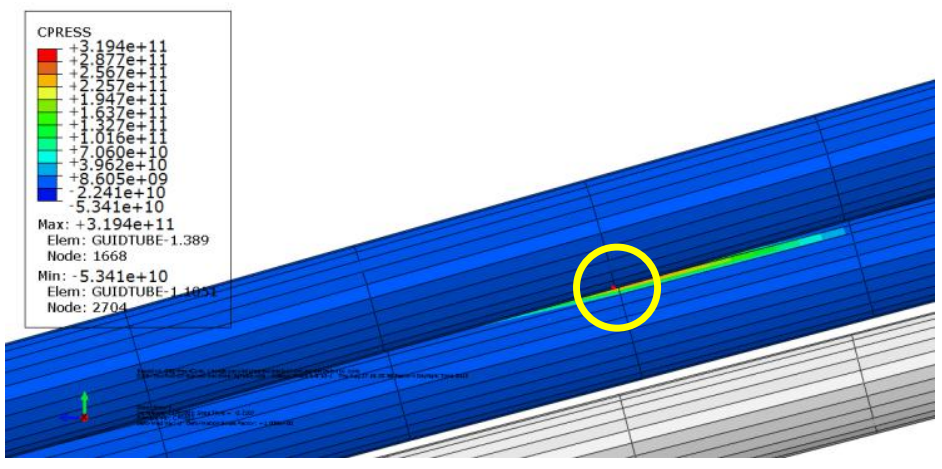

FIGURE 13. Maximum contact pressure of $3 \mathrm{~g}$ transient shock located at the contact point of guide tube and top fuel rod. 


\section{EVALUATION OF CONTACT INTERACTION BETWEEN FUEL ROD AND SPACER GRIDS INDUCED BY IMPACT LOADING}

Due to the complex design of the fuel assembly system, a simplified strategy was necessary to enable a practical solution in the FEA effort. For instance, the leaf springs and dimples of a spacer grid were modeled as translational springs in the previous dynamic simulation. Fuel rods are connected to the spacer grid through these modeled translational springs. Due to simplified spacer grid structure in SNF subassembly model, the contact interaction between the fuel rods and the spacer grids was ignored. In this section, a 3-D section model of the actual leaf spring and dimples was built to evaluate contact interaction between the fuel rod and spacer grids induced by the impact loading from cask vibration during NCT.

The illustration of a 3-D model of the fuel rod section with the dimples and leaf spring structures at the spacer grids are provided in FIGURE 14. The fuel rod length is equivalent to a quarter of the length between the spacer grids. According to the targeted spacer grid design, there are two dimples on one side of a slot and one spring on the opposite side of the slot. Each dimple and each spring is an elongated member which arches from the associated grid strap inside the associated slot. Two dimples are modeled as arch-shaped members, where the flat section of the dimple is aligned with the fuel rod's surface contour in contact. Two dimples separate along the fuel rod axial direction at a distance of $21 \mathrm{~mm}$. One spring is modeled as an arch-shaped member which is 45 degrees diagonal to the fuel rod axial orientation. The surface profile of the spacer grid region was obtained from a precision electronic scanning instrument. The sketch was implemented in ABAQUS CAE to build the dimple/spring structure geometry. Thickness for both dimples and spring was $0.47 \mathrm{~mm}$. The material of the dimple/spring is Zircaloy-4. The fuel rod dimension is provided in Adkins et al. Sept. 2013 [2] with the same material properties used in transient dynamics simulation in TABLE 1. All components were meshed with 8-node linear hexahedral brick elements. Along the thickness of dimple/spring, there were three layer meshes, and mesh size was as small as $0.16 \mathrm{~mm}$.

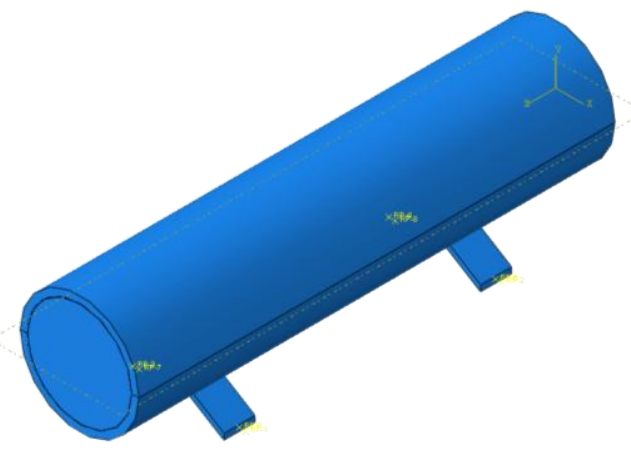

Fuel rod with two dimples

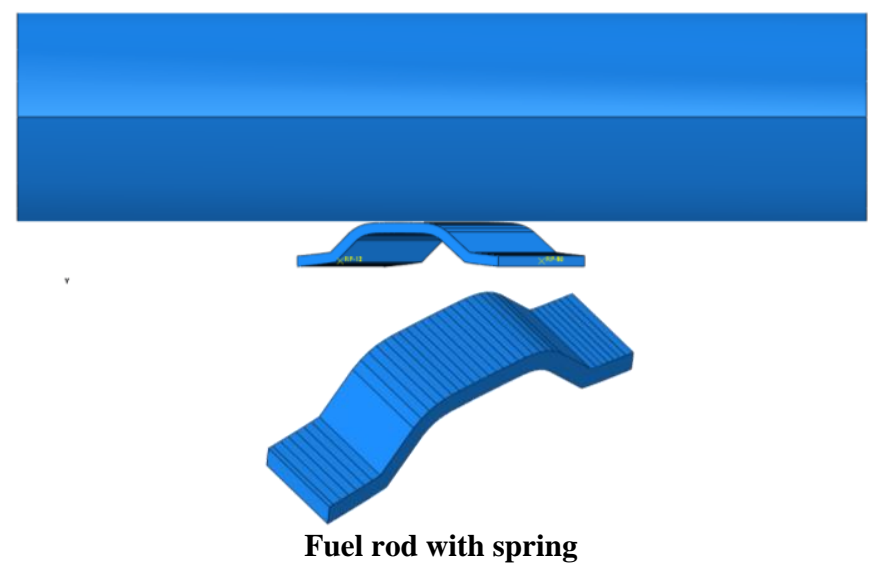

Fuel rod with spring

FIGURE 14. The 3-D model of the fuel rod section with dimples or leaf spring.

The general contact algorithm was assigned at interfaces between the cladding's bottom surface and the top surface(s) of the dimples or spring with friction coefficient 0.3. The ABAQUS dynamic explicit code was used in this impact response analysis. The approach was to simulate the impact load induced by the fuel rod inertia onto the dimples and spring within the spacer grids with a target impact velocity. Upon dynamic contact of the fuel rod and the dimples/spring, the contact stress and deformation of the interacting components (cladding, dimples, and spring) evolved progressively. Reaction forces at the contact surfaces were then estimated to evaluate the loading intensity of the target impact event. 
During the truck transportation test performed at SNL [4], the vibration load transferred from the cask to the fuel assembly has resulted in a maximum of approximately $20 \mathrm{~g}$ peak vertical vibration acceleration at the fuel rod near the spacer grid from the A7 accelerometer reading (FIGURE 15). The FEA simulation protocol was developed and carried out to generate a quantitative estimate on the loading intensity experienced by a SNF rod under such transient shock loading events. Practical assumptions were made based on the conservation of momentum and the system components' constraints to represent $20 \mathrm{~g}$ transient shock vibration periods for a fresh fuel assembly and an aging fuel assembly, including their associated initial contact velocities at the interface of the spacer grid and the fuel rod. Schematics of $20 \mathrm{~g}$ acceleration transient shock periods for fresh and aging fuel assemblies showing an increased vibration cycle period assigned for loss of system constraints in FIGURE 15. For a sound fuel assembly skeleton system, the vibration time period was assigned to $0.04 \mathrm{~s}$, and the initial contact impact velocity of the fuel rod onto the spacer grid at $20 \mathrm{~g}$ acceleration is estimated at $0.98 \mathrm{~m} / \mathrm{s}$. For an aging skeleton system, the vibration time period was assigned to $0.2 \mathrm{~s}$, and the estimated initial contact impact velocity is at $4.9 \mathrm{~m} / \mathrm{s}$. The impact response time period is strongly dependent on the structural system stiffness and is the key factor to dictate the initial contact velocity during the transient shock of NCT.

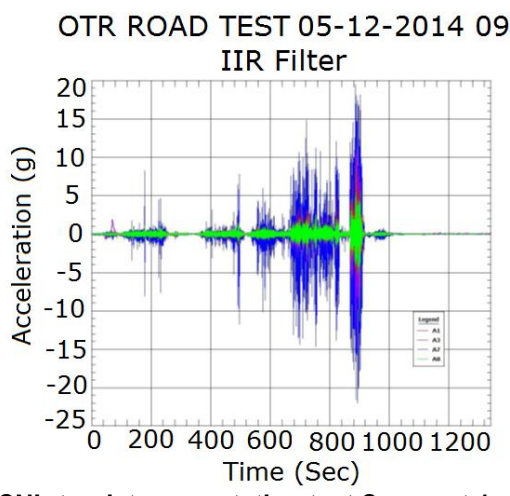

SNL truck transportation test Segment 1 accelerometer time-histories [4]

Momentum $=$ Mass $*$ Velocity $=\int F * d t, \mathrm{~F}=$ mass*acceleration

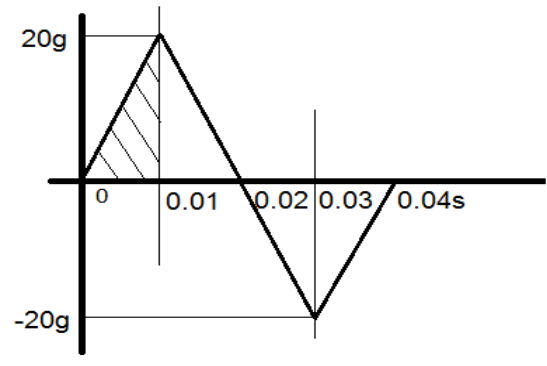

(a) Fresh fuel assembly system with good constraints among the fuel assembly skeleton system

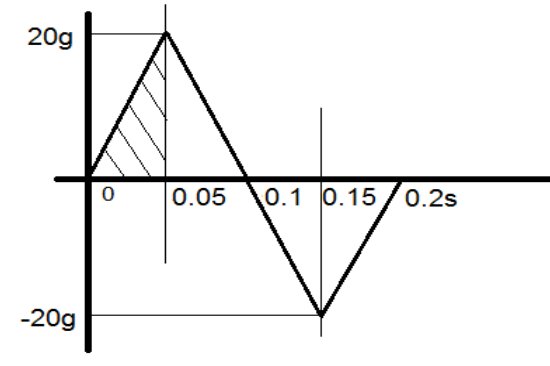

(b) Aged fuel assembly with less constraint functionality due to gaps' density increased among the fuel assembly components

FIGURE 15. SNL truck transportation test data, and schematics of $20 \mathrm{~g}$ acceleration transient shock excitations used in FEA simulations for fresh and aging fuel assemblies.

An aged fuel assembly structure would typically have lower system stiffness when compared to its initial state due to the gaps increased in the assembly's constraint skeleton system. This leads to an increase in the impact loading reaction cycle time period. To further investigate the impact contact interaction, the fuel rod and spacer grid's interaction was simulated with the dimples and leaf spring at the targeted initial contact velocity of $0.98 \mathrm{~m} / \mathrm{s}$ for a sound fuel assembly and $4.9 \mathrm{~m} / \mathrm{s}$ for an aged fuel assembly. Resulting contact stress profiles were compared to evaluate the transient shock impact effects under different scenarios.

Maximum stress occurs at dimples during the impact with the initial contact velocity of $4.9 \mathrm{~m} / \mathrm{s}$ for an aging fuel assembly, and that is shown in FIGURE 16. The maximum stress in the dimples reached the material yield strength. The significant plastic collapse of the dimples occurs under impact loading. The maximum stress on the cladding reached $386 \mathrm{MPa}$, so the cladding was not yielded. The maximum stress occurs on the inner surface of the cladding wall due to impact loading induced flexural bending tension stress on the inner wall of the cladding. FIGURE 17 shows that the contact pressure occurred on the contact surfaces of dimples and cladding during the impact at $2.19 \times 10^{-4} \mathrm{~s}$ when the dimple was starting local yielding. Contact pressure decreased as more plastic deformation occurred. Contact pressure on the dimples and cladding are $305 \mathrm{MPa}$ and $365 \mathrm{MPa}$ respectively, both of which are under material yield 
stresses. The reaction force was calculated by integrating contact pressures over the associated contact areas on the dimples. The reaction force estimated on one dimple for impact at a velocity of $4.9 \mathrm{~m} / \mathrm{s}$ is 371 $\mathrm{N}$. The total estimation on two dimples is $742 \mathrm{~N}$.
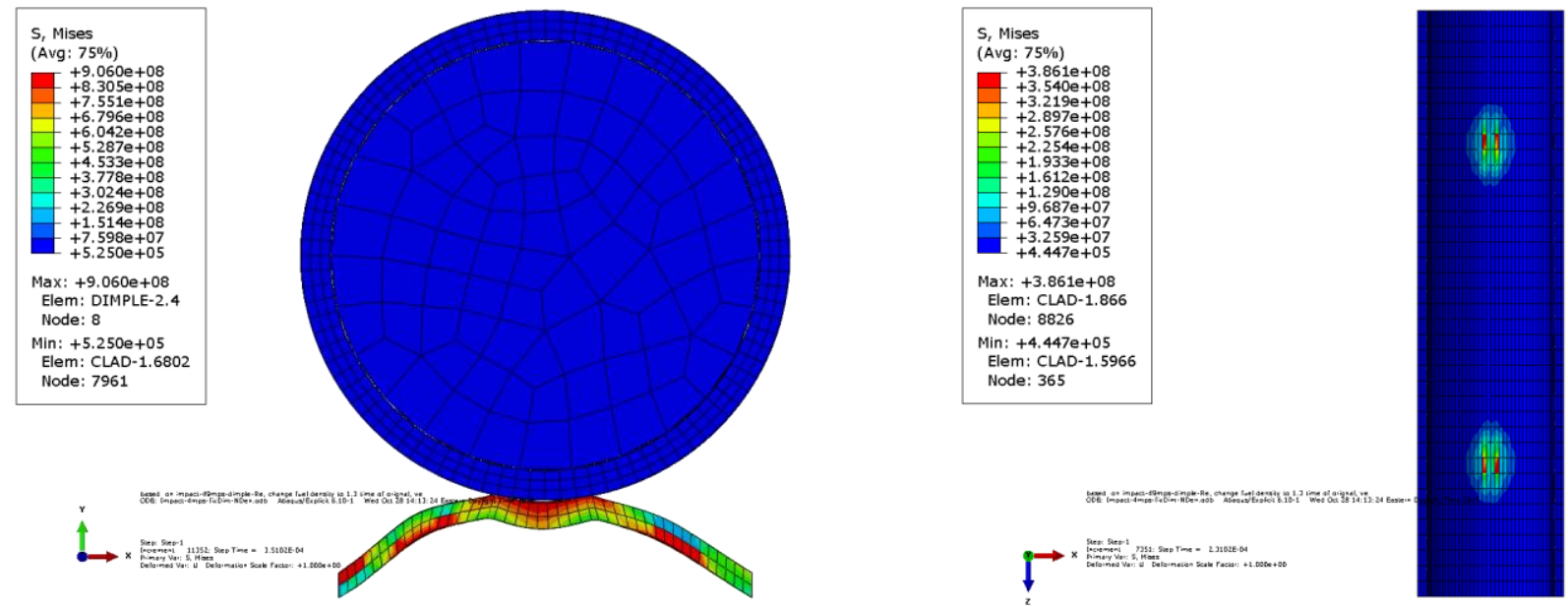

FIGURE 16. Stress distribution of dimples and cladding during fuel rod impact onto the dimples at the initial velocity of $4.9 \mathrm{~m} / \mathrm{s}$.
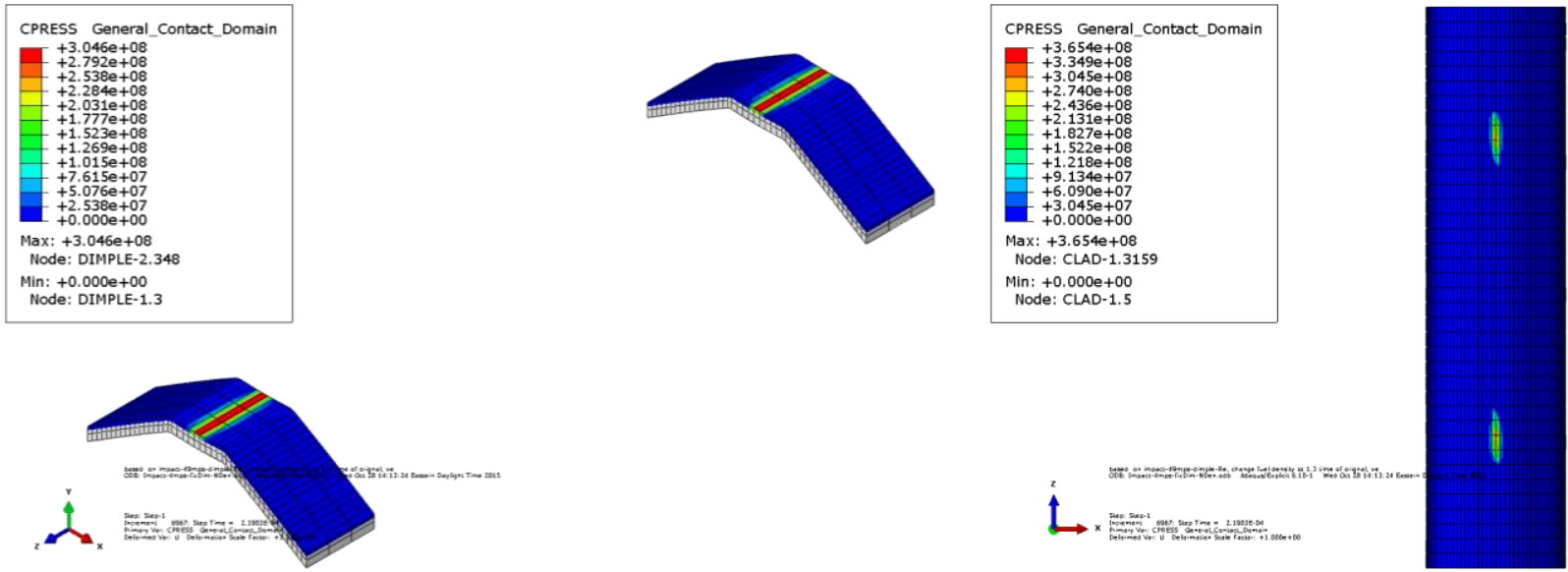

FIGURE 17. Contact pressure on dimples and cladding during a fuel rod impact on dimples at the initial velocity of $4.9 \mathrm{~m} / \mathrm{s}$.

The fuel rod's impact on the dimple with the initial contact velocity of $0.98 \mathrm{~m} / \mathrm{s}$ was investigated in the same way. The resulting contact stress profiles are much lower than that of impact velocity at $4.9 \mathrm{~m} / \mathrm{s}$. The maximum stress of $753 \mathrm{MPa}$ was reached in the dimples and is less than the yield stress. Thus, both the fuel rod and the dimples are not yielded. Under the cladding wall that is in contact with the dimples, the maximum stress of $255 \mathrm{MPa}$ was reached on the inner surface of the cladding wall. The contact pressure on the dimples of $271 \mathrm{MPa}$ and cladding of $323 \mathrm{MPa}$ are lower than that of impact at the velocity of $4.9 \mathrm{~m} / \mathrm{s}$. The contact force estimated on one dimple for impact at a velocity of $0.98 \mathrm{~m} / \mathrm{s}$ is $291 \mathrm{~N}$, which is $27 \%$ lower than that of impact speed $4.9 \mathrm{~m} / \mathrm{s}$ for an aged fuel assembly.

FIGURE 18 and FIGURE 19 show the results for the fuel rod's impact onto the spring at the initial velocity of $4.9 \mathrm{~m} / \mathrm{s}$. Similar to the fuel rod's impact on dimples at the same velocity, the spring is severely yielded during the impact event. Compared to the stress of the fuel rod's impact onto the dimples at the same velocity, the stress level of the spring is relatively lower. This is because the spring is less stiff than the dimples. This maximum clad stress of $247 \mathrm{MPa}$ is less that in the fuel rod and dimple impact case at the same impact velocity. The clads are not yielded. The maximum contact pressures on the spring and the 
clad are $298 \mathrm{MPa}$ and $191 \mathrm{MPa}$, respectively, which are under the material yield strength. The estimated contact force on the spring for impact at a velocity of $4.9 \mathrm{~m} / \mathrm{s}$ is $505 \mathrm{~N}$, which is higher than the contact force $371 \mathrm{~N}$ on one dimple for the same velocity impact. However, the reaction force on the spring is lower than the estimated total reaction force $742 \mathrm{~N}$ from both dimples.
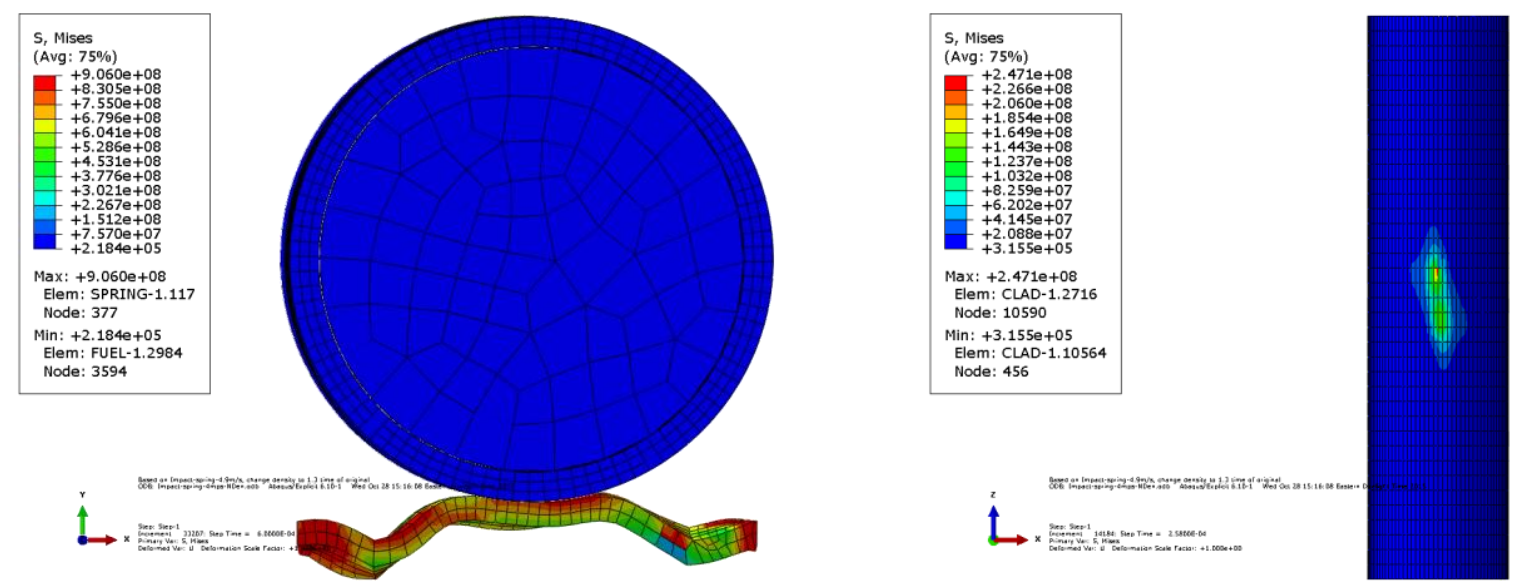

FIGURE 18. Stress distribution of the spring and cladding during the fuel rod's impact on the spring at a velocity of $4.9 \mathrm{~m} / \mathrm{s}$.
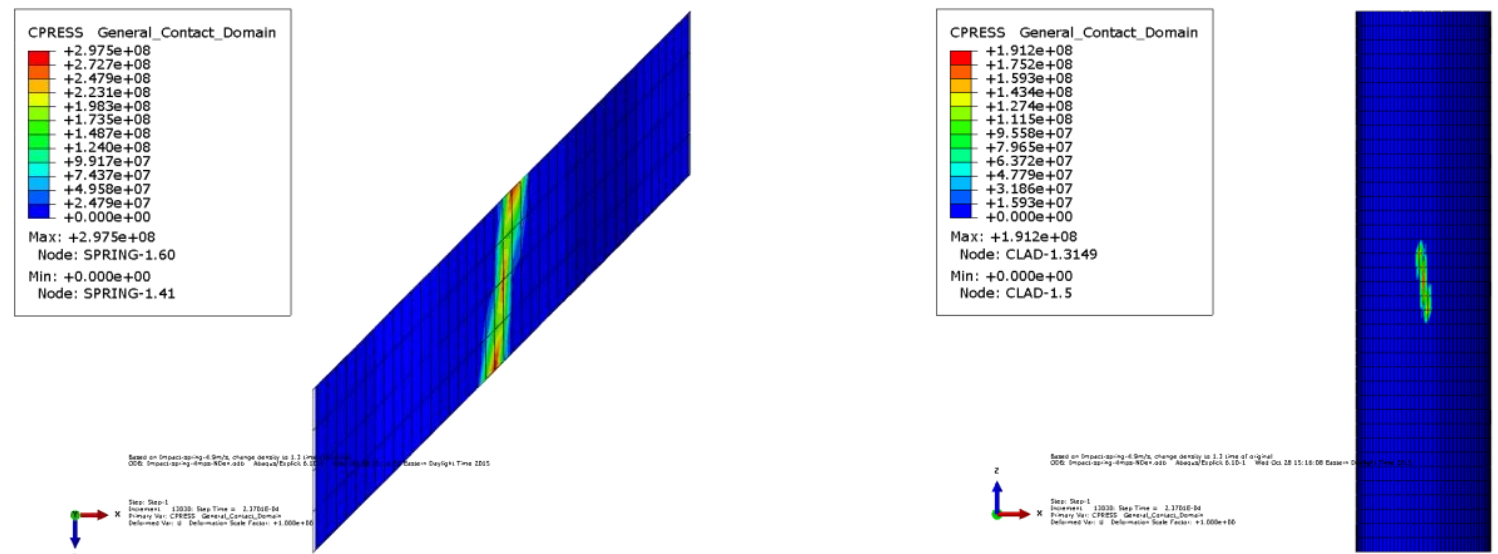

FIGURE 19. Contact pressure on the spring and cladding during the fuel rod's impact on the spring at a velocity of $4.9 \mathrm{~m} / \mathrm{s}$.

For the fuel rod's impact onto the spring at a velocity of $0.98 \mathrm{~m} / \mathrm{s}$, the maximum stresses are $702 \mathrm{MPa}$ at the spring and $151 \mathrm{MPa}$ at the clad, where both the spring and the clad are not yielded. The resultant spring contact pressure is $40 \%$ lower than that of impact speed $4.9 \mathrm{~m} / \mathrm{s}$ for an aging fuel assembly. Contact pressure on the spring of $231 \mathrm{MPa}$ is higher than that on the clad of $140 \mathrm{MPa}$. In general, the spring's contact pressure is lower than that of the dimples with the same initial contact velocity. The estimated contact force on the spring for impact at a velocity of $0.98 \mathrm{~m} / \mathrm{s}$ is $357 \mathrm{~N}$. The estimated contact forces for impact on the space grids due to $20 \mathrm{~g}$ transient shock vibration are summarized in TABLE 3.

TABLE 3. Contact intensities estimated from impact simulations of $20 \mathrm{~g}$ transient shock vibration

\begin{tabular}{|c|c|c|}
\hline Impact velocity & Contact force on spring $(\mathrm{N})$ & Contact force on dimples $(\mathrm{N})$ \\
\hline $4.9 \mathrm{~m} / \mathrm{s}$ & 505 & 742 \\
\hline $0.98 \mathrm{~m} / \mathrm{s}$ & 357 & 582 \\
\hline
\end{tabular}


The table indicates that the $4.9 \mathrm{~m} / \mathrm{s}$ contact velocity induced contact force is moderate increased compared to that of $0.98 \mathrm{~m} / \mathrm{s}$ contact velocity; which could be the results of spacer grid system structure damping and the associated structure stiffness response.

\section{COMPARISON OF STRESS INTENSITY BETWEEN TRANSIENT SHOCK LOAD AND CIRFT TESTING LOAD LEVEL}

A bridge between the transient shock loading intensity and CIRFT testing must be established to implement the transient shock loads into the CIRFT program to determine the integrated bending fatigue load with the periodic transient shock load of NCT. This capability will be used to provide an overall accumulated damage estimate or an effective SNF vibration lifetime estimate.

FIGURE 20 shows the CIRFT static bending test results and the comparison of the CIRFT test data on the H. B. Robinson (HBR) fuel rod with the moment-curvature curve converted from typical cladding stress-strain data from PNNL [21]. The estimated moment vs. curvature from PNNL data does not take fuel pellet support into consideration. For clad tubing without fuel support, the bending

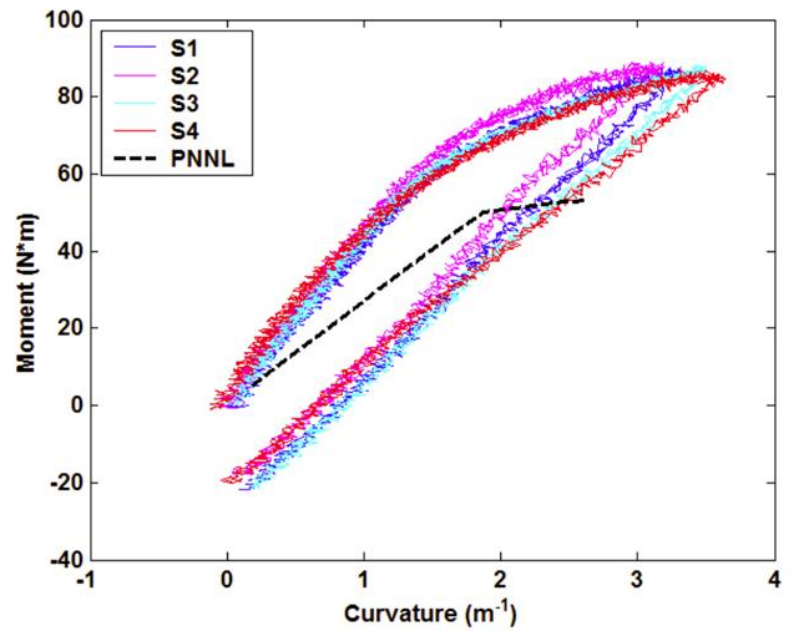

FIGURE 20. Comparison of static CIRFT test data with moment-curvature curve converted from PNNL cladding stress-strain data (without fuel)[21], S1-S4 are CIRFT test specimen names. moment resistance capacity on clad only is much less than that of the SNF rod without defueling.

To relate the stress level induced by transient shock to that of a CIRFT bending testing, the maximum clad stress of HBR SNF rod under the targeted $20 \mathrm{~g}$ transient shock load was estimated from the same FEA simulation procedure as discussed above, with the initial velocity of $4.9 \mathrm{~m} / \mathrm{s}$ for the aging fuel assembly under consideration. The HBR fuel rod dimension is the same as that provided in Adkins et al. Sept. 2013 [2]. The classic flexure normal stress formula is rearranged as shown in Eq. (1),

$$
M=\frac{\sigma I}{y}
$$

where $M$ is the bending moment, $\sigma$ is the stress on the clad, $I$ is the area moment of inertia $\left(6.666 \mathrm{E}-10 \mathrm{~m}^{4}\right)$, and $y$ is the clad radius $(5.398 \mathrm{E}-3 \mathrm{~m})$.

Equation (2) is used to determine the corresponding bending moment on the SNF rod that experienced the same stress intensity as that of the same SNF rod under $20 \mathrm{~g}$ transient shock impact loading. Here the average contact stress intensity on an HBR rod from the target $20 \mathrm{~g}$ transient shock impact load is 341 $\mathrm{MPa}$, and the estimated corresponding bending moment is $42 \mathrm{Nm}$. This moment is below the static bending moment capacity of HBR CIRFT test data $(\sim 90 \mathrm{~N}-\mathrm{m})$, and $42 \mathrm{~N}-\mathrm{m}$ is still at the linear range portion of the HBR static CIRFT test data trend. Therefore, the bending moment that has maximum bending stress matched that of a $20 \mathrm{~g}$ transient shock loading and can be effectively estimated and used to carry out high rate CIRFT vibration testing for the equivalent condition of the transient shock impact loading.

In order to estimate the damage potential of the transient shock to the SNF vibration reliability, a pilot study was carried out on Catawba (MOX) and Limerick (LMK) SNF [20]. The CIRFT specimens were

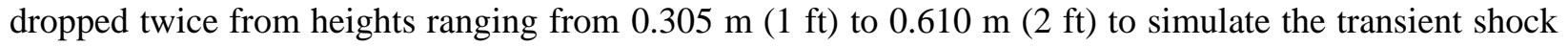


prior to carrying out the CIRFT bending cycling test at a frequency of $5 \mathrm{~Hz}$ under the target loading amplitudes. The accelerated aging of the tested mixed oxide (MOX) fuel and LMK CIRFT samples that were dropped are shown in FIGURE 21. The LMK CIRFT rods that were dropped from $0.305 \mathrm{~m}$ and then the cyclic load at $10 \mathrm{~N}-\mathrm{m}$ load show a $50 \%$ reduction in fatigue life. The MOX CIRFT rods that were dropped $0.610 \mathrm{~m}$ at $5 \mathrm{~N}-\mathrm{m}$ load show a $73 \%$ reduction in fatigue life. The reduction in the lifetime of CIRFT-tested SNF samples was observed by combining the harmonic vibration loading and the dropinduced transient shock load [21].

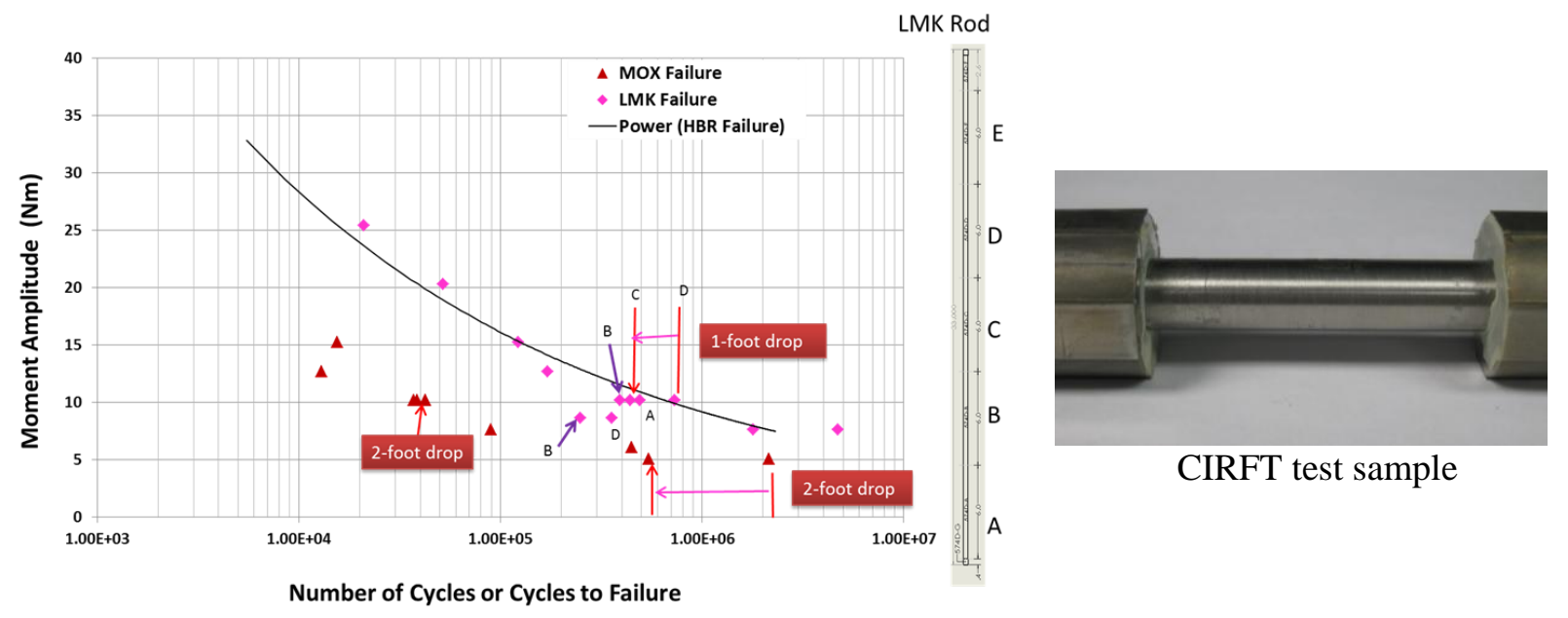

FIGURE 21. Pilot transient shocks test on MOX and LMK SNF [21].Power is power-law curve fitting of HBR CIRFT data. A-E marked the locations where the CIRFT test specimens were cut from LMK rod.

This pilot test shows that the drop-induced transient shock load impacts the performance of the SNF CIRFT test samples. In order to understand the drop intensity, the FEA simulation was conducted to investigate the contact reaction at the CIRFT test sample during the drop impact loading events. FIGURE 21 also shows a CIRFT test sample with a $0.0508 \mathrm{~m}$ gage section and two rigid gripping sections on both ends. In the FEA, a SNF Zr-4 rod was modeled as test sample. The total weight of the simulated testing sample, including the $0.0508 \mathrm{~m}$ gauge section and the two end grip sections, is $310 \mathrm{~g}$. The SNF CIRFT samples were dropped from heights of 0.305-0.610 m (1-2 ft.) onto a stainless steel hollow square block stand $(0.0127 \mathrm{~m} \times 0.0254 \mathrm{~m})$ with a wall thickness of $0.0038 \mathrm{~m}$. For the free fall, the velocity of the falling object when it comes to impact the object on the ground is calculated as

$$
v=\sqrt{2 g h}
$$

Where $g$ is gravity $\left(9.8 \mathrm{~m} / \mathrm{s}^{2}\right)$ and $h$ is the drop distance between the drop starting point to the top surface of the stainless block. When the sample was dropped from $0.305 \mathrm{~m}(1 \mathrm{ft}$.), it hit the stainless steel block at a speed of $2.444 \mathrm{~m} / \mathrm{s}$ upon the contact. The ABAQUS impact simulation procedure was applied to investigate the contact reaction of the fuel rod under the impact induced by the drop.

FIGURE 22 shows the stress profiles in the fuel rod where the maximum stress on the clad at contact locations is $348 \mathrm{MPa}$. As in the previous impact study, the maximum stress occurs on the inner surface of the cladding wall due to impact-induced bending. This maximum stress is less than the material yield strength of $906 \mathrm{MPa}$. Nevertheless, residual stress contours remain in the cladding after the impact event and will eventually reduce the fatigue life of the sample additional fatigue loading, as validated by the CIRFT tests illustrated in FIGURE 21. FIGURE 23 shows that the highest contact pressure occurred during the CIRFT rod's impact to the stainless steel block stand. The maximum contact pressure on clad is $260 \mathrm{MPa}$, which is under the material yield strength. The reaction force estimated from the contact pressure profiles is $641 \mathrm{~N}$. Compared to the similar case on two dimples under impact induced by $20 \mathrm{~g}$ transient shock for aging fuel assembly, the contact force was lower than that estimated $742 \mathrm{~N}$ reaction 
force on two dimples. Therefore, the CIRFT sample $0.305 \mathrm{~m}$ drop-induced impact did not reach the estimated intensity of a $20 \mathrm{~g}$ transient shock from FEA.
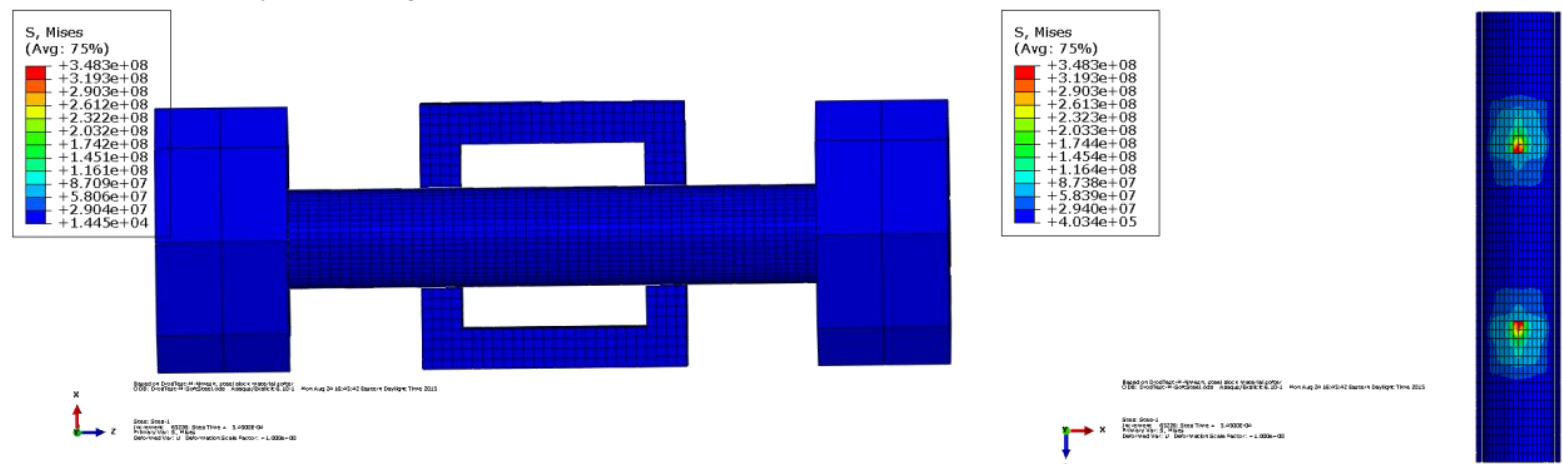

FIGURE 22. Stress on stainless steel block and cladding under impact induced by a $0.305 \mathrm{~m}$ drop of the CIRFT sample.
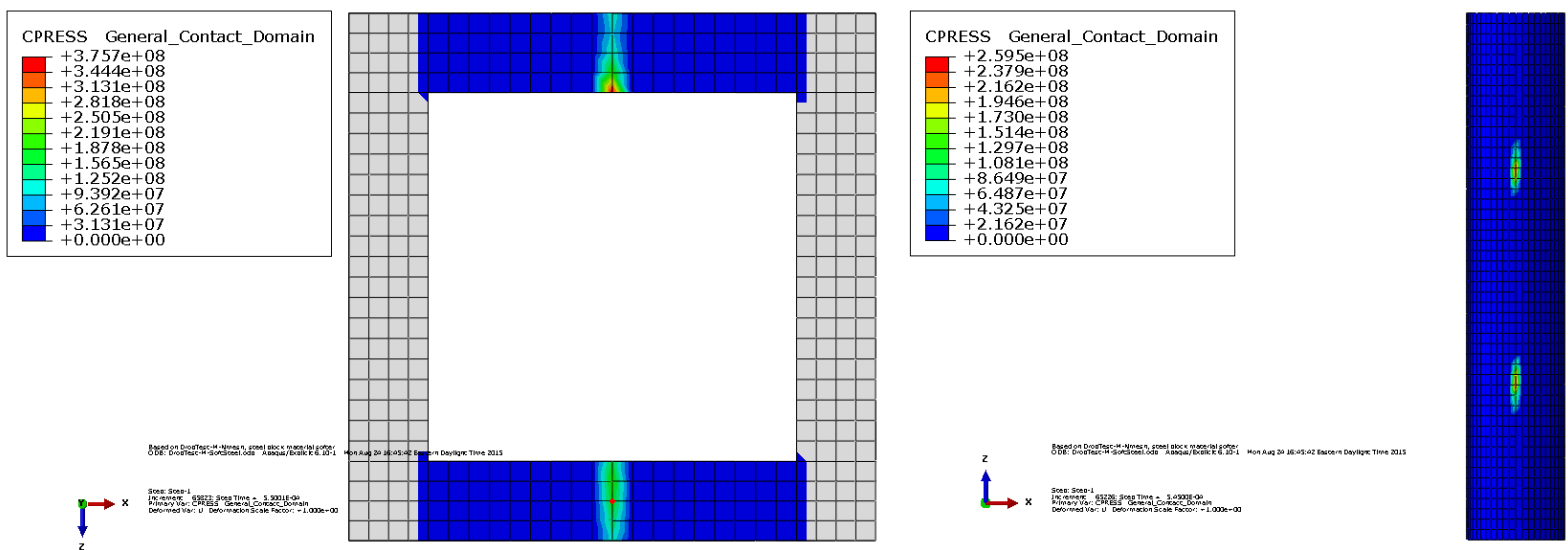

FIGURE 23. Contact pressure on stainless steel block and cladding under impact induced by a $0.305 \mathrm{~m}$ drop of CIRFT sample.

\section{SUMMARY}

An SNF was simplified as a subassembly model with two fuel rods, a guide tube, and the associated spacer grids to resemble the fuel rods located at the bottom edge of the full fuel assembly. The guide tube was on top of two fuel rods. Each fuel rod had 240 fuel pellets and one cladding $3.658 \mathrm{~m}$ long. The partial basket wall, eight spacer grids, top and bottom nozzles were included in the subassembly model.

Modal analysis was performed on the SNF subassembly model in the frequency domain. The results provided more information on the frequency characteristics of the fuel subassembly model. The natural frequency of the dominant mode of the subassembly model is much lower than the modal analysis results for a cradle/cask system model and a concrete block of equivalent mass with the basket/assembly weight model $[11,12]$. Thus, system models with different structure detail levels have significantly different natural frequency characteristics.

In the time domain, the transient dynamic simulation consists of two stages: (1) an initialization stage to establish contact due to gravity, and (2) the second stage for transient dynamic loading. During the second stage, the acceleration as a sine wave form or impulse signal to represent normal transportation excitation or shock excitation for transient dynamic analysis. The dynamic response of the fuel subassembly model in the initialization stage shows that intermediate spacer grids rest on the basket floor after sagging due to gravity, but the top and bottom spacer grids do not. The maximum stress occurs at the guide tube, and 
there is no yielding in the system. The maximum contact pressure occurs at the location where the intermediate spacer is touching the basket, and the pressure level is low.

A $0.5 \mathrm{~g}$ sine wave acceleration excitation represents normal transportation vibration. The dynamic responses of fuel subassembly model under the vibration of $0.5 \mathrm{~g}$ sine wave acceleration shows that system stress level is higher than that in the initial stage. After normal transportation vibration, the fuel rod, guide tube, spacer grids and basket wall did not deform as uniformly as in the initialization stage. Some intermediate spacer grids lost contact with the basket wall. From von Mises stress distribution, besides the fuel pellets, the max stress occurs at the bottom Inconel spacer, where the material yields. The maximum stress in the guide tube has reached the yield strength. Yielding on the guide tube occurs at both top and bottom nozzle locations. Results show that the guide tube and spacer grids are at the risk of failure, which is consistent with the consideration of fuel assembly damage evaluation in the EPRI report [3]. Due to the horizontal orientation for transport, the guide tubes and spacer grids become the major components bearing the gravity and dynamic loads for the fuel assembly. The integrity of the guide tube and spacer grids critically affects the vibration modes and the vibration intensity of fuel assembly during transport.

Under vibration of $0.5 \mathrm{~g}$ sine wave acceleration, the maximum stress of the claddings is under material yield strength. The basket wall and top and bottom nozzles were yield; and maximum contact pressure occurs at the location where the spacer grid touches the basket floor. The maximum contact pressure level is almost one order magnitude higher than it was in the initialization stage, but it is still relatively low.

A $3 \mathrm{~g}$ impulse acceleration excitation represents transient shock during transportation. As in the dynamic response under $0.5 \mathrm{~g}$ sine wave acceleration excitation, yielding occurs at the bottom Inconel spacers. The guide tube also yielded at the top and bottom nozzle locations during $3 \mathrm{~g}$ transient shock excitation. Thus there were concerns for the integrity of the spacer grids and the guide tube under transient shock loading during NCT. The maximum stress level on the cladding was twice of that under $0.5 \mathrm{~g}$ sine wave acceleration excitation near yield strength. The basket and top and bottom nozzles had reached yield strength. The maximum contact pressure occurred at the location where the guide tube contacts the top clad. The maximum contact pressure level is high at this contacting moment. An issue of concern is that this dynamic contact interaction might form another transient shock loading to the fuel assembly.

To further evaluate the contact's interaction intensity between the fuel rod and the spacer grids induced by impact loading, a 3-D finite element (FE) section model of the detailed leaf spring/dimples has been developed to simulate the impact between the fuel rod and the spacer grids as induced by the cask vibration during transportation. Under the target $20 \mathrm{~g}$ transient shock, the spring/dimples severely yielded during the high velocity impact. The contact forces on the spring/dimples estimated from the FEA were high enough to cause significant plastic deformation. The maximum stress occurred on the inner surface of the cladding wall because the bending induced by the impact caused tension there. The equivalent bending moment of $42 \mathrm{~N}-\mathrm{m}$, estimated from the resultant stress on the clad under $20 \mathrm{~g}$ transient shock, can be used as the loading in CIRFT vibration testing for estimating the SNF fatigue life induced by the associated periodic transient shocks.

The pilot drop testing of the SNF CIRFT samples showed that the impact induced by the drop affected the integrity of the tested samples. An FEA simulation was conducted to investigate the contact intensity of the CIRFT test sample during impact. Results from the simulated $0.305 \mathrm{~m}$ drop of the CIRFT sample revealed that the maximum stress on the clad at contact locations was less than half of material yield strength. However, the residual stress in the cladding affected the fatigue lifetime of SNF CIRFT testing samples. The contact force was lower than that estimated from $20 \mathrm{~g}$ transient shock on the spacer grid and 
fuel clad. Therefore, the CIRFT sample with $0.305 \mathrm{~m}$ drop impact did not reach the estimated intensity of a $20 \mathrm{~g}$ transient shock.

The SNF subassembly model can be further expanded to apply to a $17 \times 17$ full fuel assembly. A fullscale model of the targeted fuel assembly will provide more realistic fuel assembly system interactions and their associated boundary conditions, resulting in a more reliable dynamic response prediction outcome. Refinement of the model can also provide more insights on the transient shock loading effect to the SNF's vibration reliability with increased confidence.

\section{ACKNOWLEDGMENTS}

This research was sponsored by the DOE Used Fuel Disposition Campaign (UFDC) under DOE contract DE-AC05-00OR22725 with UT-Battelle, LLC. Authors thank Hong Wang for CIRFT testing data, Nicholas Klymyshyn for valuable discussion, and program managers Bruce Bevard and John Scaglione for their support and guidance during the program development.

\section{REFERENCES}

1. Adkins, H. E. et al., US Department of Energy, Used Fuel Disposition Campaign, Used Nuclear Fuel Loading and Structural Performance under Normal Conditions of Transport-Modeling, Simulation and Experimental Integration RD\&D Plan, FCRD-UFD-2013-000135 (April 1, 2013).

2. Adkins, H. E. et al., US Department of Energy, Used Fuel Disposition Campaign, Used Nuclear Fuel Loading and Structural Performance under Normal Conditions of Transport-Demonstration of Approach and Results of Used Fuel Performance Characterization (September 30, 2013).

3. Rashid, J. Spent Fuel Transportation Applications-Assessment of Cladding Performance, EPRI a Synthesis Report-1015048 (December 2007).

4. McConnell, P., Wauneka, R., Saltzstein, S., and Sorenson, K. US Department of Energy Used Fuel Disposition Campaign, Normal Conditions of Transport Truck Test of a Surrogate Fuel Assembly, FCRD-UFD-2014-000066, Sandia National Laboratories (August 29, 2014).

5. Geelhood, K., Adkins, H., Sanborn, S., Koeppel, B., and Klymyshyn, N. "Analytical Model Methodology Development and Demonstration of Approach on Used Fuel Performance Characterization for Condition of Normal Transportation," 14568, WM2014 Conference, Phoenix, Arizona, USA (March, 2014).

6. Bignell, J., Flores, G., and Ammerman, D. "Cask Assembly Level Modeling to Determine Used Nuclear Fuel Assembly Loading Environments Resulting From Normal Conditions of Rail Transport," 14511, WM2014 Conference, Phoenix, Arizona, USA (March, 2014).

7. Sanborn, S., Koeppel, B., Klymyshyn, N., Adkins, H., and Geelhood, K., "Assembly Level Modeling and Transportation Damage Prediction of Used Nuclear Fuel (UNF) Cladding," 14569, WM2014 Conference, Phoenix, Arizona, USA (March, 2014).

8. Coleman, J. L., and Spears, R. E., "Detailed PWR Fuel Rod and Grid Finite Element Analysis to Provide Equivalent Rod Stiffness and Damping and Equivalent Grid Shell Thickness to PWR Used Nuclear Fuel (UNF) Assembly," 14525, WM2014 Conference, Phoenix, Arizona, USA (March) 2014

9. Chakraborty, P., et al., Modeling and Simulation of Used Nuclear Fuel during Transportation with Consideration of Hydride Effects and Cyclic Fatigue, FCRD-UFD-2015-000273, INL/EXT-15-36697 (September 30, 2015).

10. Rashid, J., Wong, F., and Dunham, R., Spent-Fuel Transportation Applications-Normal Conditions of Transport, EPRI Report-1015049 (June 2007). 
11. Ross, S., Klymyshyn, N., Jensen, P., Best, R., Maheras, S., McConnell, P., and Orchard, J., "Rail Shock and Vibration Pre-Test Modeling of a Used Nuclear Fuel Assembly," IHLRWM 2015, Charleston, SC (April 12-16, 2015).

12. Ross, S. B., et al., Used Fuel Rail Shock and Vibration Testing Options Analysis, FCRD-UFD-2014000327, PNNL-23709 (September 25, 2014).

13. Wang, H., Wang, J.-A.J., Tan, T., Jiang, H., Cox, T., Howard, R., Bevard, B., and Flanagan, M., "Development of U-Frame Bending System for Studying the Vibration Integrity of Spent Nuclear Fuel," Journal of Nuclear Material 440: 201-13 (2013).

14. Wang, J.-A.J., Wang, H., and Tan, T., An Innovative Dynamic Reversal Bending Fatigue Testing System for Evaluating Spent Nuclear Fuel Rod Vibration Integrity or Other Materials Fatigue Aging Performance, ORNL Invention Disclosure 201102593, DOE S 124,149, April 8, 2011, Patent in review, 13/396,413 (February 14, 2012).

15. Wang, J.-A.J., Wang, H., Tan, T., Jiang, H., Cox, T., and Yan, Y., Progress Letter Report on U Frame Test Setup and Bending Fatigue Test for Vibration Integrity Study (Out-of-Cell Fatigue Testing Development-Task 2.2), ORNL/TM-2011/531, Oak Ridge National Laboratory, Oak Ridge, TN (January 2012).

16. BARC Highlights, "Nuclear Fuel Cycle," Chapter 4, Bhabha Atomic Research Center, Trombay, Mumbai, India, 35-39. (2007) http://www.barc.ernet.in/publications/eb/golden/nfc/toc/Chapter\%204/4.6.pdf

17. Geelhood, K. J., et al., PNNL Stress/Strain Correlation for Zircaloy, PNNL-17700, Pacific Northwest National Laboratory, Richland, Washington (July 2008).

18. Special Metals INCONEL @ Alloy 718, http://www.matweb.com (accessed November 13, 2015).

19. Product Data Bulletin, 304/304L stainless steel, AK Steel Corporation. <http://www.aksteel.com>.

20. Special Metals T300 series stainless steel, http://www.matweb.com (accessed November 13, 2015).

21. Wang, J.-A.J., Wang, H., Jiang, H., Yan, Y., and Bevard, B., FY 2015 Status Report: CIRFT Testing of High-Burnup Used Nuclear Fuel Rods from the Pressurized Water Reactor and Boiling Water Reactor Environments, ORNL/SPR-2015/313, Oak Ridge National Laboratory, Oak Ridge, Tennessee (2015). 